\title{
On the maximum likelihood estimation of the ToA under an imperfect path loss exponent
}

\author{
Isabel Valera ${ }^{1 *}$, Bamrung Tau Sieskul ${ }^{2}$ and Joaquín Míguez ${ }^{1}$
}

\begin{abstract}
We investigate the estimation of the time of arrival (TOA) of a radio signal transmitted over a flat-fading channel. The path attenuation is assumed to depend only on the transmitter-receiver distance and the path loss exponent (PLE) which, in turn, depends on the physical environment. All previous approaches to the problem either assume that the PLE is perfectly known or rely on estimators of the ToA which do not depend on the PLE. In this paper, we introduce a novel analysis of the performance of the maximum likelihood (ML) estimator of the ToA under an imperfect knowledge of the PLE. Specifically, we carry out a Taylor series expansion that approximates the bias and the root mean square error of the ML estimator in closed form as a function of the PLE error. The analysis is first carried out for a path loss model in which the received signal gain depends only on the PLE and the transmitter-receiver distance. Then, we extend the obtained results to account also for shadow fading scenarios. Our computer simulations show that this approximate analysis is accurate when the signal-to-noise ratio (SNR) of the received signal is medium to high. A simple Monte Carlo method based on the analysis is also proposed. This technique is computationally efficient and yields a better approximation of the ML estimator in the low SNR region. The obtained analytical (and Monte Carlo) approximations can be useful at the design stage of wireless communication and localization systems.
\end{abstract}

Keywords: Time-of-arrival estimation, Maximum likelihood estimator, Path loss exponent

\section{Introduction}

The estimation of a signal time of arrival (ToA), also called time delay, plays an important role in applied signal processing problems, e.g., synchronization [1], array processing [2], tracking and positioning of mobile terminals [3-8], or even bioengineering [9]. Let us focus on the estimation of the ToA in wireless radio links. In [10], two simple (and practically appealing) estimators of the ToA are studied: the maximum correlation (MC) and the maximum likelihood (ML) estimators. The MC estimator of the ToA depends only on the correlation between the transmitted and the received signals. The ML estimator of the ToA, on the other hand, takes also into account the path attenuation, which depends on the distance between the transmitter and the receiver, and the path loss exponent (PLE) of the environment. The performances of both estimators in mobile positioning applications are analyzed in [8], where it is shown that the ML estimator attains a

*Correspondence: ivalera@tsc.uc3m.es

${ }^{1}$ Department of Signal Theory and Communications, University Carlos III of Madrid, Madrid, Spain

Full list of author information is available at the end of the article better accuracy when the PLE is perfectly known a priori. However, the latter assumption is often unrealistic for a practical scenario, because the PLE may change according to variations in the environment and thus may need to be estimated. Tracking the fluctuations of the PLE is specially important in problems that involve the localization of mobile terminals, since the existing positioning techniques based on the ToA estimation are extremely sensitive to errors in the PLE [6].

The problem of dealing with unknown PLEs has been addressed in several related works. In [7], a positioning application with several receivers and one transmitter is considered. The PLEs are assumed to be different and random, with either uniform or normal distributions. The availability of different PLEs for each link increases the localization accuracy compared to the identical PLE assumption. In $[11,12]$, the PLE is estimated from the measurements, whereas in [13-15], several algorithms for the PLE estimation are proposed. The authors of [14] describe three distributed algorithms for PLE estimation in large wireless networks in the presence of node location uncertainties, $m$-Nakagami fading and interference.

\section{是 Springer}

(c) 2013 Valera et al: licensee Springer. This is an Open Access article distributed under the terms of the Creative Commons Attribution License (http://creativecommons.org/licenses/by/2.0), which permits unrestricted use, distribution, and reproduction in any medium, provided the original work is properly cited. 
In [13] and [15], the algorithms for the estimation of the path loss inside a sensor network are designed using previous path loss measurements among sensors. Specifically, in [15], the PLEs are estimated for each node applying the ML criterion and using both ToA and received-signalstrength measurements among the sensors. In [16], a handover algorithm is presented using the least squares estimate of the path loss parameters for each link from a mobile station to a base station. In $[17,18]$, the sensitivity of the ML estimator of the direction of arrival (DoA) of a received signal under model error, i.e., with a mismatch in the PLE, is investigated. To our best knowledge, the problem of estimating the ToA with both the path attenuation and the PLE unknown has not been tackled yet.

The goal of this paper is to investigate the performance of the ML estimator of the ToA under an imperfect PLE. Specifically, we aim at obtaining analytical or semianalytical approximations of the bias and the mean square error (MSE) of the ML estimator, both given in terms of the PLE error and the signal-to-noise ratio (SNR) of the communication channel. Such approximations are intended to be useful in the design and setup of the communication and localization wireless systems, as they may considerably alleviate the need for a lengthy and computationally expensive simulation of the whole system.

This article is organized as follows. We first describes the signal model and then briefly reviews the expressions of the MC and the ML estimators of the ToA with perfect knowledge of the PLE in Section 2, including formulas for their MSE performances. In Section 3, we carry out an approximate analysis of the bias and the MSE attained by the ML estimator of the ToA under imperfect PLE. We first apply the method in [18] to study the error between the ML joint estimator of the PLE and the ToA, on one hand, and the ML estimator of the ToA under an imperfect knowledge of the PLE, on the other hand. The difficulty of this study is that the resulting error depends on the ML joint estimates of the ToA and the PLE, which are random variables related to the noise. In order to tackle this limitation and provide an analytical (albeit approximate) expression for the error between the true and the estimated ToA, we analyze the ML estimator of the ToA with mismatched PLE in the second part of Section 3. In particular, we propose a new method to analyze the estimation error based on the Taylor series expansion. In Section 4, we investigate the extension of these results to shadow fading environments [19]. In particular, we first show that the approximate bias is insensitive to the shadow fading, whereas the MSE becomes a random variable (the probability density function (pdf) of which is obtained). Then, we provide a straightforward algorithm to obtain a Monte Carlo estimate of the MSE by drawing only from simple Gaussian distributions (instead of simulating the whole transmission system). In Section 5, we present illustrative computer simulation results based on the transmission of an ultra-wideband (UWB) signal. We have chosen UWB signaling to validate our analysis because it provides an excellent means for wireless positioning due to its high resolution in the time domain [4]. Finally, Section 6 is devoted to the conclusions.

\section{Signal model}

\subsection{Received signal}

Consider a wireless transmission link with one single transmitter and one single receiver. Following [20], the received signal can be expressed as

$$
r(t)=a s(t-\tau)+n(t)
$$

where $r(t)$ is the received signal, $s(t)$ is the transmitted signal, which is assumed deterministic and possibly complexvalued, $a \in \mathbb{R}^{+}$and $\tau \geq 0$ are the path gain and the propagation delay (commonly referred to as ToA) between the transmitter and the receiver, and $n(t)$ is the additive noise at the receiver, assumed to be a circularly-symmetric complex-valued zero-mean white Gaussian process with double-sided power spectral density $\sigma_{\mathrm{n}}^{2}$. In (1), $\tau$ is the parameter to be estimated.

Assume that the received signal $r(t)$ is observed in the interval $\left(0, T_{o}\right]$, and let $E_{\mathrm{S}}=\int_{0}^{T_{0}}|s(t)|^{2} \mathrm{~d} t$ be the energy of the transmitted signal in that period. Then, the relation between the transmitted and the received signal energies in terms of the path gain can be written as

$$
E_{\mathrm{r}}=a^{2} E_{\mathrm{s}}
$$

In the literature, the path gain $a$ is often modeled as a deterministic parameter (see, e.g., [21-29]). However, it can be elaborated in a more precise way to include the propagation effect $[8,10]$. In this paper, we assume a combined path loss and log-normal shadowing model. The received power in $\mathrm{dB}$ is given by (see, e.g., [19], p. 45)

$$
P_{\mathrm{r}}(d B)=P_{\mathrm{t}}+10 \log _{10}(\kappa)-10 \gamma \log _{10}\left(d / d_{0}\right)-\psi_{\mathrm{dB}},
$$

where $P_{\mathrm{t}}$ is the transmitted power in $\mathrm{dB}, d_{0}$ is the closein distance in the far-field region; $d=c \tau$ is the distance between the transmitter and the receiver ( $c$ is the speed of light), $\gamma$ is the PLE at the base station, $\psi_{\mathrm{dB}}$ is a Gaussian random variable with zero mean and variance $\sigma_{\mathrm{dB}}^{2}$ which characterizes the shadow fading (see, e.g., [19], p. 45 and [30], p. 139), and $\kappa$ is a unitless constant depending on the antenna characteristics and the average channel attenuation given by

$$
\kappa=\frac{c^{2}}{16 \pi^{2} d_{0}^{2} f_{0}^{2}}
$$


with $f_{0}$ being the central frequency of the transmitted signal. If we convert the power in Equation (3) into natural units, then the received energy can also be written as

$$
E_{\mathrm{r}}=E_{\mathrm{s}} \frac{\kappa}{\psi}\left(\frac{d_{0}}{d}\right)^{\gamma}
$$

where the random factor $\psi=10 \frac{\psi_{\mathrm{dB}}}{10}$ is due to the shadow fading. Substituting (2) into (5), we obtain an explicit expression for the path gain $a$ as

$$
a=\sqrt{\frac{\kappa}{\psi}}\left(\frac{d_{0}}{c \tau}\right)^{\frac{1}{2} \gamma}
$$

Let us remark that, since the shadow fading term $\psi_{\mathrm{dB}}$ is normally distributed, the path gain $a$ of Equation (6) is a random variable. For the sake of simplicity, in the analysis of the ML estimator performance in Section 3, we ignore the effect of the shadow fading by taking the expectation with respect to (w.r.t.) $\psi$ of $P_{\mathrm{r}}$ in (3). As a consequence, the path gain becomes deterministic and reduces to

$$
a=\sqrt{\kappa}\left(\frac{d_{0}}{c \tau}\right)^{\frac{1}{2} \gamma}
$$

which coincides with the expression of the path gain already used in $[8,10]$. The shadow fading effect is taken explicitly into account in Section 4.

\section{2 $M C$ and $M L$ estimators of the ToA}

In this subsection, we briefly review the MC and ML estimators of the ToA. In the literature, the PLE $\gamma$ is assumed known, and the effect of the shadow fading is ignored; hence, the path gain is deterministic, known and given by (7) [10].

Let us further use the notation $\Re(\cdot)$ and $(\cdot)^{*}$ as the real part and the conjugate of a complex number, respectively. The MC and ML estimators of the ToA are given by [10]

$$
\begin{aligned}
& \hat{\tau}_{\mathrm{MC}}=\arg \max _{\tau} \rho(\tau), \\
& \hat{\tau}_{\mathrm{ML}}=\arg \min _{\tau} f_{\mathrm{ML}}(\tau),
\end{aligned}
$$

respectively, where $\rho(\tau)=\int_{0}^{T_{\mathrm{o}}} \mathfrak{R}\left(r(t) s^{*}(t-\tau)\right) \mathrm{d} t$ is the correlation between the transmitted and the received signals, and the function $f_{\mathrm{ML}}(\tau)=a^{2} E_{\mathrm{s}}-2 a \rho(\tau)$ is the loglikelihood ${ }^{\mathrm{a}}$ of $\tau$ given $r(t)$ [10]. Note that $a$ is a function of the propagation delay, as shown in (7). For conciseness, this dependence is left implicit throughout the paper.

In [10] the authors obtain approximate formulas for the errors (the bias and the MSE) of the MC and the ML estimators of the ToA, the latter under perfect knowledge of the PLE. In particular, the values of the MSE for the MC and the ML estimators can be approximated, respectively, as [10]

$$
\begin{aligned}
\mathrm{MSE}_{\mathrm{MC}} & \approx \frac{1}{8 \pi^{2} \frac{S}{N} a^{2} \bar{\beta}^{2}}, \\
\mathrm{MSE}_{\mathrm{ML}} & \approx \frac{1}{\frac{S}{N} a^{2}\left(8 \pi^{2} \bar{\beta}^{2}+\frac{\gamma^{2}}{2 \tau^{2}}\right)},
\end{aligned}
$$

where $\tau$ and $\gamma$ are, respectively, the true values of the propagation delay and the path gain; the path loss exponent $(\bar{\beta})$ is the effective bandwidth, and $\frac{S}{N}=\frac{E_{\mathrm{s}}}{\sigma_{\mathrm{n}}^{2}}$ is the signal-to-noise ratio (see [10] for additional details). Note that the expression in Equation (9b) coincides with the Cramér-Rao bound (see, e.g., [23-29]) for the time delay estimation [10].

\section{Performance analysis}

In this section, we analyze the performance of ML estimator of the ToA, $\tau$, subject to a model error. In particular, we assume that the knowledge of the PLE is not exact. This is a common situation in practice, since different phenomena, such as the movement of the transmitter (or the receiver) and sudden changes in the weather, directly cause changes in the PLE from one observation period to the next. In order to model the uncertainty in the nominal value of the PLE, $\gamma$, consider an additive perturbation

$$
\gamma=\gamma_{0}+\delta_{\gamma},
$$

where $\gamma_{0}$ is the (unknown) true value of the PLE, and $\delta_{\gamma}$ is an (equally unknown) additive error. In this section, we assume that the path gain has the form shown in (7); therefore, the following analysis is valid for environments with no shadow fading.

The $\log$-likelihood $f_{\mathrm{ML}}$ in (8b) implicitly depends on the PLE, because the path gain $a$ in (7) is a function of $\gamma$. Since we assume a mismatch between the true value of the PLE, $\gamma_{0}$, and the value available at the receiver, $\gamma$, we need to make this dependence explicit in order to analyze the estimator performance. Therefore, let us write the $\log$-likelihood $f_{\mathrm{ML}}$ as a function of two real variables, the propagation delay $\tau$ and the nominal PLE $\gamma$

$$
\begin{aligned}
f_{\mathrm{ML}}(\tau, \gamma) & =a^{2} E_{\mathrm{s}}-2 a \rho(\tau) \\
& =\kappa\left(\frac{d_{0}}{c \tau}\right)^{\gamma} E_{\mathrm{s}}-2 \sqrt{\kappa}\left(\frac{d_{0}}{c \tau}\right)^{\frac{1}{2} \gamma} \rho(\tau),
\end{aligned}
$$

where the second equality is obtained by expanding the path gain $a$, as given in Equation (7) (see also Equations (32) to (35) in [10] for further details on the derivation of the log-likelihood function $f_{\mathrm{ML}}$ ). Recall that $\rho(\tau)=\int_{0}^{T_{\mathrm{o}}} \Re\left(r(t) s^{*}(t-\tau)\right) \mathrm{d} t$ is the correlation between the transmitted and the received signals, and $E_{\mathrm{s}}$ is the energy of the transmitted signal. Figure 1 illustrates the shape of the $\log$-likelihood function $f_{\mathrm{ML}}$. Note 


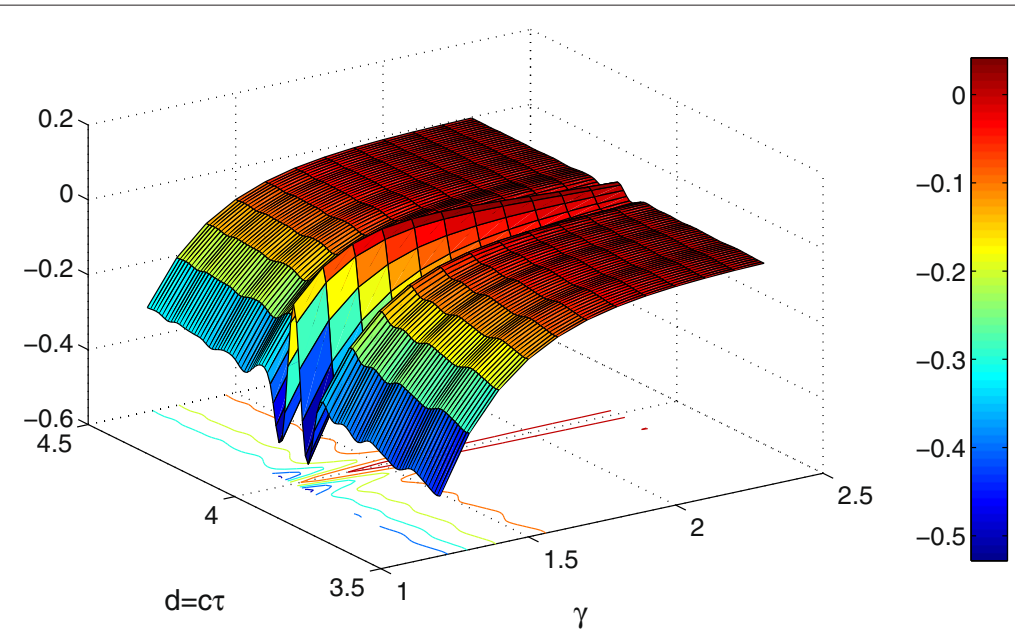

Figure 1 Sample realization of $-\boldsymbol{f}_{\mathbf{M L}}$. Parameters are set to $\gamma_{0}=1.7, c \tau_{0}=4 \mathrm{~m}, \mathrm{SNR}=30 \mathrm{~dB}, \bar{\beta}=3.1007 \times 10^{9} \mathrm{~Hz}$, and sampling time of $0.01 \mathrm{ps}$. The global maximum is located at the true values of the TOA and the PLE.

that this is just a sample realization of $-f_{\mathrm{ML}}$, since its actual form depends on the specific communication system and the received data. The resulting ML estimator of the ToA $\tau$ as a function of the nominal PLE $\gamma$ is

$$
\hat{\tau}_{\mathrm{ML}}(\gamma)=\arg \min _{\tau} f_{\mathrm{ML}}(\tau, \gamma)
$$

In former works, the sensitivity analysis of the ML estimator under model error has been investigated for DoA estimation (see, e.g., $[17,18])$. Similar ideas can be applied to investigate the mis-modeled estimation problem in (12), as shown in Section 3.1. This approach suffers from several limitations, though. For this reason, we introduce a novel approximate analysis based on a Taylor series expansion in Section 3.2.

\subsection{Friedlander's method}

In this subsection, we adapt the methodology proposed in [18] to the problem of the ToA estimation. Note that, while our objective in the present paper is the characterization of the ToA estimation error under an imperfect PLE, in [18], the author addresses the DoA estimation for a sensor array under the imperfect knowledge of the channel parameters. Therefore, the log-likelihood function is different for each of the two estimation problems (DoA and ToA).

Let $\hat{\tau}_{\mathrm{ML}}$ and $\hat{\gamma}_{\mathrm{ML}}$ denote the ML estimates of the ToA and the PLE, respectively, which jointly maximize the likelihood function, i.e., assume that $\left.\frac{\partial}{\partial \tau} f_{\mathrm{ML}}(\tau, \gamma)\right|_{\begin{array}{c}\tau=\hat{\tau}_{\mathrm{ML}} \\ \gamma=\hat{\gamma}_{\mathrm{ML}}\end{array}}=0$ and $\left.\frac{\partial}{\partial \gamma} f_{\mathrm{ML}}(\tau, \gamma)\right|_{\substack{\tau=\hat{\tau}_{\mathrm{ML}} \\ \gamma=\hat{\gamma}_{\mathrm{ML}}}}=0$. Following [18], we approxi- mate the error between $\hat{\tau}_{\mathrm{ML}}$ and the ML estimate of the ToA under a mismatch in the PLE, denoted by $\hat{\tau}_{\mathrm{ML}}(\gamma)$, as

$$
\hat{\tau}_{\mathrm{ML}}(\gamma)-\hat{\tau}_{\mathrm{ML}} \approx-\frac{\left.\left(\gamma-\gamma_{\mathrm{ML}}\right) \frac{\partial^{2}}{\partial \gamma \partial \tau} f_{\mathrm{ML}}(\tau, \gamma)\right|_{\substack{\tau=\hat{\tau}_{\mathrm{ML}} \\
\gamma=\hat{\gamma}_{\mathrm{ML}}}}}{\left.\frac{\partial^{2}}{\partial \tau^{2}} f_{\mathrm{ML}}(\tau, \gamma)\right|_{\begin{array}{l}
\tau=\hat{\tau}_{\mathrm{ML}} \\
\gamma=\hat{\gamma}_{\mathrm{ML}}
\end{array}}}
$$$$
=\varepsilon_{F}\left(\hat{\tau}_{\mathrm{ML}}, \hat{\gamma}_{\mathrm{ML}}\right) \text {, }
$$

which is obtained by approximating $\frac{\partial}{\partial \tau} f_{\mathrm{ML}}(\tau, \gamma)$ by its first order Taylor series expansion around $\hat{\tau}_{\mathrm{ML}}$ and $\hat{\gamma}_{\mathrm{ML}}$ (see [18], Section III).

In order to find an explicit form of 13, we can elaborate both the numerator and the denominator of $\varepsilon_{F}\left(\hat{\tau}_{\mathrm{ML}}, \hat{\gamma}_{\mathrm{ML}}\right)$ as

$$
\begin{aligned}
& \left.\frac{\partial^{2}}{\partial \tau^{2}} f_{\mathrm{ML}}(\tau, \gamma)\right|_{\substack{\tau=\hat{\tau}_{\mathrm{ML}} \\
\gamma=\hat{\gamma}_{\mathrm{ML}}}}=\left(\frac{1}{\tau^{2}} \gamma(1+\gamma) E_{\mathrm{s}} a^{2}-\frac{1}{\tau^{2}} \gamma\right. \\
& \times\left(1+\frac{1}{2} \gamma\right) a \rho(\tau)+\frac{1}{\tau} 2 \gamma a \frac{\partial}{\partial \tau} \rho(\tau) \\
& \left.-2 a \frac{\partial^{2}}{\partial \tau^{2}} \rho(\tau)\right)\left.\right|_{\substack{\tau=\hat{\tau}_{\mathrm{ML}} \\
\gamma=\hat{\gamma}_{\mathrm{ML}}}} \\
& =\frac{1}{\hat{\tau}_{\mathrm{ML}}^{2}} \hat{\gamma}_{\mathrm{ML}}\left(1+\hat{\gamma}_{\mathrm{ML}}\right) E_{\mathrm{s}} a_{\mathrm{ML}}^{2}-\frac{1}{\hat{\tau}_{\mathrm{ML}}^{2}} \hat{\gamma}_{\mathrm{ML}} \\
& \times\left(1+\frac{1}{2} \hat{\gamma}_{\mathrm{ML}}\right) a_{\mathrm{ML}} \rho\left(\hat{\tau}_{\mathrm{ML}}\right) \\
& +\frac{1}{\hat{\tau}_{\mathrm{ML}}} 2 \hat{\gamma}_{\mathrm{ML}} a_{\mathrm{ML}} \dot{\rho}\left(\hat{\tau}_{\mathrm{ML}}\right)-2 a_{\mathrm{ML}} \ddot{\rho}\left(\hat{\tau}_{\mathrm{ML}}\right)
\end{aligned}
$$


and

$$
\begin{aligned}
& \left.\frac{\partial^{2}}{\partial \tau \partial \gamma} f_{\mathrm{ML}}(\tau, \gamma)\right|_{\substack{\tau=\hat{\mathrm{T}}_{\mathrm{ML}} \\
\gamma=\hat{\gamma}_{\mathrm{ML}}}}=\left(\frac{1}{\tau} a\left(\rho(\tau)-E_{\mathrm{s}} a\right)+\frac{1}{\tau} \gamma\right. \\
& \times \ln \left(\frac{d_{0}}{c \tau}\right) a\left(\frac{1}{2} \rho(\tau)-E_{\mathrm{s}} a\right) \\
& \left.-\ln \left(\frac{d_{0}}{c \tau}\right) a \frac{\partial}{\partial \tau} \rho(\tau)\right)\left.\right|_{\substack{\tau=\hat{\tau}_{\mathrm{ML}} \\
\gamma=\hat{\gamma}_{\mathrm{ML}}}} \\
& =\frac{1}{\hat{\tau}_{\mathrm{ML}}} a_{\mathrm{ML}}\left(\rho\left(\hat{\tau}_{\mathrm{ML}}\right)-E_{\mathrm{s}} a_{\mathrm{ML}}\right) \\
& +\frac{1}{\hat{\tau}_{\mathrm{ML}}} \hat{\gamma}_{\mathrm{ML}} \ln \left(\frac{d_{0}}{c \hat{\tau}_{\mathrm{ML}}}\right) a_{\mathrm{ML}} \\
& \times\left(\frac{1}{2} \rho\left(\hat{\tau}_{\mathrm{ML}}\right)-E_{\mathrm{s}} a_{\mathrm{ML}}\right) \\
& -\ln \left(\frac{d_{0}}{c \hat{\tau}_{\mathrm{ML}}}\right) a_{\mathrm{ML}} \dot{\rho}\left(\hat{\tau}_{\mathrm{ML}}\right),
\end{aligned}
$$

where $a_{\mathrm{ML}}=a\left(\hat{\tau}_{\mathrm{ML}}, \hat{\gamma}_{\mathrm{ML}}\right)$ is the value of the path gain for $\tau=\hat{\tau}_{\mathrm{ML}}$ and $\gamma=\hat{\gamma}_{\mathrm{ML}} ; \rho(\tau), \dot{\rho}(\tau)$, and $\ddot{\rho}(\tau)$ are correlation functions given by

$$
\rho(\tau)=\int_{0}^{T_{\mathrm{o}}} \Re\left(r(t) s^{*}(t-\tau)\right) \mathrm{d} t=a_{0} \rho_{\mathrm{ss}}(\tau)+\rho_{\mathrm{ns}}(\tau),
$$

$$
\begin{aligned}
\dot{\rho}(\tau) & =\int_{0}^{T_{\mathrm{o}}} \Re\left(r(t) \frac{\partial}{\partial \tau} s^{*}(t-\tau)\right) \mathrm{d} t \\
& =a_{0} \frac{\partial}{\partial \tau} \rho_{\mathrm{ss}}(\tau)+\frac{\partial}{\partial \tau} \rho_{\mathrm{ns}}(\tau), \\
\ddot{\rho}(\tau) & =\int_{0}^{T_{\mathrm{o}}} \Re\left(r(t) \frac{\partial^{2}}{\partial \tau^{2}} s^{*}(t-\tau)\right) \mathrm{d} t \\
& =a_{0} \frac{\partial^{2}}{\partial \tau^{2}} \rho_{\mathrm{ss}}(\tau)+\frac{\partial^{2}}{\partial \tau^{2}} \rho_{\mathrm{ns}}(\tau),
\end{aligned}
$$

respectively, where $a_{0}=a\left(\tau_{0}, \gamma_{0}\right)$ is the true value of the path gain, and $\rho_{\mathrm{ss}}(\tau)$ is the autocorrelation of the transmitted signal, which is given by

$$
\rho_{\mathrm{ss}}(\tau)=\int_{0}^{T_{\mathrm{o}}} \Re\left(s\left(t-\tau_{0}\right) s^{*}(t-\tau)\right) \mathrm{d} t
$$

and $\rho_{\mathrm{ns}}(\tau)$ is the correlation between the noise and the transmitted signal, i.e.,

$$
\rho_{\mathrm{ns}}(\tau)=\int_{0}^{T_{\mathrm{o}}} \Re\left(n(t) s^{*}(t-\tau)\right) \mathrm{d} t
$$

Substituting (14) and (15) into (13), we obtain a closed formula for the (approximate) estimation error, namely,

$$
\begin{aligned}
\varepsilon_{F}\left(\hat{\tau}_{\mathrm{ML}}, \hat{\gamma}_{\mathrm{ML}}\right)= & \frac{-\left(\gamma-\gamma_{\mathrm{ML}}\right)}{h\left(\hat{\tau}_{\mathrm{ML}}\right)} \times\left[\frac{1}{\hat{\tau}_{\mathrm{ML}}^{2}} \hat{\gamma}_{\mathrm{ML}}\left(1+\hat{\gamma}_{\mathrm{ML}}\right) E_{\mathrm{s}} a_{\mathrm{ML}}\right. \\
& -\frac{1}{\hat{\tau}_{\mathrm{ML}}^{2}} \hat{\gamma}_{\mathrm{ML}}\left(1+\frac{1}{2} \hat{\gamma}_{\mathrm{ML}}\right) \rho\left(\hat{\tau}_{\mathrm{ML}}\right) \\
& \left.+\frac{1}{\hat{\tau}_{\mathrm{ML}}} 2 \hat{\gamma}_{\mathrm{ML}} \dot{\rho}\left(\hat{\tau}_{\mathrm{ML}}\right)-2 \ddot{\rho}\left(\hat{\tau}_{\mathrm{ML}}\right)\right]
\end{aligned}
$$

where

$$
\begin{aligned}
h\left(\hat{\tau}_{\mathrm{ML}}\right)= & \frac{1}{\hat{\tau}_{\mathrm{ML}}}\left(\rho\left(\hat{\tau}_{\mathrm{ML}}\right)-E_{\mathrm{s}} a_{\mathrm{ML}}\right)+\frac{1}{\hat{\tau}_{\mathrm{ML}}} \hat{\gamma}_{\mathrm{ML}} \ln \left(\frac{d_{0}}{c \hat{\tau}_{\mathrm{ML}}}\right) \\
& \times\left(\frac{1}{2} \rho\left(\hat{\tau}_{\mathrm{ML}}\right)-E_{\mathrm{S}} a_{\mathrm{ML}}\right)-\ln \left(\frac{d_{0}}{c \hat{\tau}_{\mathrm{ML}}}\right) \dot{\rho}\left(\hat{\tau}_{\mathrm{ML}}\right) .
\end{aligned}
$$

Friedlander's approach provides us with a characterization of the error between two estimators of the ToA, namely, the 'full' ML estimator obtained by maximizing the likelihood function $-f_{\mathrm{ML}}(\tau, \gamma)$ jointly over $\tau$ and $\gamma$, on one hand, and the conditional ML estimator obtained for a fixed (but imperfect or mismatched) value of the PLE $\gamma$, denoted by $\hat{\tau}_{\mathrm{ML}}(\gamma)$, on the other. However, the error given in (19) is a function of the random variables $\hat{\tau}_{\mathrm{ML}}, \hat{\gamma}_{\mathrm{ML}}, \rho\left(\hat{\tau}_{\mathrm{ML}}\right), \dot{\rho}\left(\hat{\tau}_{\mathrm{ML}}\right)$, and $\ddot{\rho}\left(\hat{\tau}_{\mathrm{ML}}\right)$, which in turn, depend on the realization of the noise process $n(t)$. This dependence makes the derivation of the first and the second moments of $\varepsilon_{F}\left(\hat{\tau}_{\mathrm{ML}}, \hat{\gamma}_{\mathrm{ML}}\right)$ analytically intractable.

To tackle this limitation, we propose a different approach that aims at characterizing the error between the estimator $\hat{\tau}_{\mathrm{ML}}(\gamma)$ for a fixed imperfect PLE, $\gamma$, and the true value of the ToA, $\tau_{0}$. Our analysis not only leads to a mathematically tractable approximation but it is also, in our opinion, more meaningful from a practical perspective.

\subsection{Performance analysis based on a Taylor series expansion}

In this section, we evaluate the error between the true and the estimated values of the ToA. Let $\tau_{0}$ and $\gamma_{0}$ denote the true values of the ToA and the PLE, respectively, both assumed deterministic. The first-order Taylor series expansion of the partial derivative $\frac{\partial}{\partial \tau} f_{\mathrm{ML}}(\tau, \gamma)$ in two 
variables around the true values $\tau_{0}$ and $\gamma_{0}$ can be written as (see, e.g., [31-33])

$$
\begin{aligned}
\frac{\partial}{\partial \tau} f_{\mathrm{ML}}(\tau, \gamma)= & \left.\frac{\partial}{\partial \tau} f_{\mathrm{ML}}(\tau, \gamma)\right|_{\substack{\tau=\tau_{0} \\
\gamma=\gamma_{0}}}+\left.\frac{\partial^{2}}{\partial \tau \partial \tau} f_{\mathrm{ML}}(\tau, \gamma)\right|_{\substack{\tau=\tau_{0} \\
\gamma=\gamma_{0}}} \\
& \times\left(\tau-\tau_{0}\right)+\left.\frac{\partial^{2}}{\partial \tau \partial \gamma} f_{\mathrm{ML}}(\tau, \gamma)\right|_{\substack{\tau=\tau_{0} \\
\gamma=\gamma_{0}}} \\
& \times\left(\gamma-\gamma_{0}\right)+o\left(\left(\tau-\tau_{0}\right)^{2}+\left(\gamma-\gamma_{0}\right)^{2}\right) .
\end{aligned}
$$

Assuming a large SNR, the terms of the second or higher order in $o\left(\left(\hat{\tau}_{\mathrm{ML}}(\gamma)-\tau_{0}\right)^{2}+\left(\gamma-\gamma_{0}\right)^{2}\right)$ are negligible [25], p.236). The estimated value $\tau=\hat{\tau}_{\mathrm{ML}}(\gamma)$ minimizes $f_{\mathrm{ML}}(\tau, \gamma)$ using a mis-modeled PLE, i.e., $\hat{\tau}_{\mathrm{ML}}(\gamma)$ is a solution to the equation $\left.\frac{\partial}{\partial \tau} f_{\mathrm{ML}}(\tau, \gamma)\right|_{\tau=\hat{\tau}_{\mathrm{ML}}(\gamma)}=0$. Then, by dropping the term $o\left(\left(\hat{\tau}_{\mathrm{ML}}(\gamma)-\tau_{0}\right)^{2}+\left(\gamma-\gamma_{0}\right)^{2}\right)$, we can approximate the error between the true and the estimated ToA as

$$
\begin{aligned}
& \hat{\tau}_{\mathrm{ML}}(\gamma)-\tau_{0} \\
& \approx-\frac{\left.\frac{\partial}{\partial \tau} f_{\mathrm{ML}}(\tau, \gamma)\right|_{\substack{\tau=\tau_{0} \\
\gamma=\gamma_{0}}}}{\left.\left.\frac{\partial^{2}}{\partial \tau^{2}} f_{\mathrm{ML}}(\tau, \gamma)\right|_{\substack{\tau=\tau_{0} \\
\gamma=\gamma_{0}}} \frac{\partial^{2}}{\partial \tau \partial \gamma \gamma} f_{\mathrm{ML}}(\tau, \gamma)\right|_{\substack{\tau=\tau_{0} \\
\gamma=\gamma_{0}}}} \\
& =\tilde{\varepsilon}_{\mathrm{TE}}\left(\tau_{0}, \gamma\right) .
\end{aligned}
$$

In order to provide analytical expressions for the bias and the MSE of the error in (22), we introduce a further approximation into the error $\tilde{\varepsilon}_{\mathrm{TE}}\left(\tau_{0}, \gamma\right)$ by following [23], p. 642, Equation (17-9.6) as

$$
\begin{aligned}
& \tilde{\varepsilon}_{\mathrm{TE}}\left(\tau_{0}, \gamma\right) \\
& \approx-\frac{\left.\frac{\partial}{\partial \tau} f_{\mathrm{ML}}(\tau, \gamma)\right|_{\substack{\tau=\tau_{0} \\
\gamma=\gamma_{0}}}+\left.\left(\gamma-\gamma_{0}\right) \frac{\partial^{2}}{\partial \tau \partial \gamma} f_{\mathrm{ML}}(\tau, \gamma)\right|_{\substack{\tau=\tau_{0} \\
\gamma=\gamma_{0}}}}{\mathrm{E}_{n(t)}\left\{\left.\frac{\partial^{2}}{\partial \tau^{2}} f_{\mathrm{ML}}(\tau, \gamma)\right|_{\substack{\tau=\tau_{0} \\
\gamma=\gamma_{0}}}\right\}}
\end{aligned}
$$$$
=\varepsilon_{\mathrm{TE}}\left(\tau_{0}, \gamma\right)
$$

where the expectation in the denominator is introduced for mathematical tractability. The derivatives $\frac{\partial}{\partial \tau} f_{\mathrm{ML}}(\tau, \gamma)$ and $\frac{\partial^{2}}{\partial \gamma \partial \tau} f_{\mathrm{ML}}(\tau, \gamma)$ for $\tau=\tau_{0}$ and $\gamma=\gamma_{0}$ in (23) can be evaluated explicitly and yield

$$
\left.\frac{\partial}{\partial \tau} f_{\mathrm{ML}}(\tau, \gamma)\right|_{\substack{\tau=\tau_{0} \\ \gamma=\gamma_{0}}}=\frac{1}{\tau_{0}} \gamma_{0} a_{0} \rho_{\mathrm{ns}, 0}-2 a_{0} \dot{\rho}_{\mathrm{ns}, 0},
$$

and

$$
\begin{aligned}
\left.\frac{\partial^{2}}{\partial \gamma \partial \tau} f_{\mathrm{ML}}(\tau, \gamma)\right|_{\substack{\tau=\tau_{0} \\
\gamma=\gamma_{0}}}= & \frac{1}{\tau_{0}} a_{0} \rho_{\mathrm{ns}, 0}-\frac{1}{2 \tau_{0}} \gamma_{0} a_{0}\left(E_{\mathrm{s}} a_{0}-\rho_{\mathrm{ns}, 0}\right) \\
& \times \ln \left(\frac{d_{0}}{c \tau_{0}}\right)-a_{0} \dot{\rho}_{\mathrm{ns}, 0} \ln \left(\frac{d_{0}}{c \tau_{0}}\right),
\end{aligned}
$$

respectively, where $\rho_{\mathrm{ns}, 0}=\rho_{\mathrm{ns}}\left(\tau_{0}\right)$ and $\dot{\rho}_{\mathrm{ns}, 0}=$ $\left.\frac{\partial}{\partial \tau} \rho_{\mathrm{ns}}(\tau)\right|_{\substack{\tau=\tau_{0} \\ \gamma=\gamma_{0}}}$ Additionally, the expected value of $\frac{\partial^{2}}{\partial \tau^{2}} f_{\mathrm{ML}}(\tau, \gamma)$ evaluated at $\tau=\tau_{0}$ and $\gamma=\gamma_{0}$ is

$$
\mathrm{E}_{n(t)}\left\{\left.\frac{\partial^{2}}{\partial \tau^{2}} f_{\mathrm{ML}}(\tau, \gamma)\right|_{\substack{\tau=\tau_{0} \\ \gamma=\gamma_{0}}}\right\}=\frac{1}{2 \tau_{0}^{2}} \gamma_{0}^{2} E_{\mathrm{s}} a_{0}^{2}+8 \pi^{2} \bar{\beta}^{2} E_{\mathrm{s}} a_{0}^{2},
$$

where $\bar{\beta}$ is the effective bandwidth. Equations (24), (25), and (26) are explicitly obtained in Appendix 1. Then, substituting (26), (25), and (24) into (23), we obtain the (approximate) closed formula for the ToA estimation error

$$
\begin{aligned}
\varepsilon_{\mathrm{TE}}\left(\tau_{0}, \gamma\right)= & -\frac{1}{\frac{1}{2 \tau_{0}^{2}} \gamma_{0}^{2} E_{\mathrm{s}}+8 \pi^{2} \bar{\beta}^{2} E_{\mathrm{s}}}\left[\frac{1}{\tau_{0}} \gamma_{0} \rho_{\mathrm{ns}, 0}-2 \dot{\rho}_{\mathrm{ns}, 0}\right. \\
& +\left(\gamma-\gamma_{0}\right)\left(\frac{1}{\tau_{0}} \rho_{\mathrm{ns}, 0}-\frac{1}{2 \tau_{0}} \gamma_{0}\left(E_{\mathrm{s}} a_{0}-\rho_{\mathrm{ns}, 0}\right)\right. \\
& \left.\left.\times \ln \left(\frac{d_{0}}{c \tau_{0}}\right)-\dot{\rho}_{\mathrm{ns}, 0} \ln \left(\frac{d_{0}}{c \tau_{0}}\right)\right)\right] .
\end{aligned}
$$

The bias of the conditional ML estimator given an imperfect PLE $\gamma$ is obtained by taking the expectation of (23), i.e.,

$$
\begin{aligned}
& \mathrm{E}_{n(t)}\left\{\varepsilon_{\mathrm{TE}}\left(\tau_{0}, \gamma\right)\right\} \\
& =-\frac{\mathrm{E}_{n(t)}\left\{\left.\frac{\partial}{\partial \tau} f_{\mathrm{ML}}(\tau, \gamma)\right|_{\substack{\tau=\tau_{0} \\
\gamma=\gamma_{0}}}\right\}+\left(\gamma-\gamma_{0}\right) \mathrm{E}_{n(t)}\left\{\left.\frac{\partial^{2}}{\partial \tau \partial \gamma} f_{\mathrm{ML}}(\tau, \gamma)\right|_{\substack{\tau=\tau_{0} \\
\gamma=\gamma_{0}}}\right\}}{\frac{1}{2 \tau_{0}^{2}} \gamma_{0}^{2} E_{\mathrm{s}} a_{0}^{2}+8 \pi^{2} \bar{\beta}^{2} E_{\mathrm{s}} a_{0}^{2}} .
\end{aligned}
$$

The expectations $\mathrm{E}_{n(t)}\left\{\left.\frac{\partial}{\partial \tau} f_{\mathrm{ML}}(\tau, \gamma)\right|_{\substack{\tau=\tau_{0} \\ \gamma=\gamma_{0}}}\right\}$ and $\mathrm{E}_{n(t)}\left\{\frac{\partial^{2}}{\partial \tau \partial \gamma}\right.$ $\left.\left.f_{\mathrm{ML}}(\tau, \gamma)\right|_{\substack{\tau=\tau_{0} \\ \gamma=\gamma_{0}}}\right\}$ can be readily calculated by taking into account that $\mathrm{E}_{n(t)}\left\{\rho_{\mathrm{ns}, 0}\right\}=0$ and $\mathrm{E}_{n(t)}\left\{\dot{\rho}_{\mathrm{ns}, 0}\right\}=0$, and yield

$$
\begin{aligned}
\mathrm{E}_{n(t)}\left\{\left.\frac{\partial}{\partial \tau} f_{\mathrm{ML}}(\tau, \gamma)\right|_{\substack{\tau=\tau_{0} \\
\gamma=\gamma_{0}}}\right\} & =0, \\
\mathrm{E}_{n(t)}\left\{\left.\frac{\partial^{2}}{\partial \tau \partial \gamma} f_{\mathrm{ML}}(\tau, \gamma)\right|_{\substack{\tau=\tau_{0} \\
\gamma=\gamma_{0}}}\right\} & =-\frac{1}{2 \tau_{0}} \gamma_{0} a_{0}^{2} E_{\mathrm{s}} \ln \left(\frac{d_{0}}{c \tau_{0}}\right) .
\end{aligned}
$$


Hence, by substituting (29a) and (29b) into (28), the bias of $\hat{\tau}_{\mathrm{ML}}(\gamma)$ can be written as

$$
\mathrm{E}_{n(t)}\left\{\varepsilon_{\mathrm{TE}}\left(\tau_{0}, \gamma\right)\right\}=\left(\gamma-\gamma_{0}\right) \frac{\gamma_{0} \tau_{0} \ln \left(\frac{d_{0}}{c \tau_{0}}\right)}{\gamma_{0}^{2}+16 \pi^{2} \bar{\beta}^{2} \tau_{0}^{2}},
$$

which vanishes when $\gamma=\gamma_{0}$, i.e., when the PLE is perfectly known.

The MSE of the estimator $\hat{\tau}_{\mathrm{ML}}(\gamma)$ can also be obtained as a function of the nominal (imperfect) PLE $\gamma$, namely

$$
\begin{aligned}
& \mathrm{E}_{n(t)}\left\{\varepsilon_{\mathrm{TE}}^{2}\left(\tau_{0}, \gamma\right)\right\} \\
& =\frac{\mathrm{E}_{n(t)}\left\{\left(\left.\frac{\partial}{\partial \tau} f_{\mathrm{ML}}(\tau, \gamma)\right|_{\substack{\tau=\tau_{0} \\
\gamma=\gamma_{0}}}+\left.\left(\gamma-\gamma_{0}\right) \frac{\partial^{2}}{\partial \tau \partial \gamma} f_{\mathrm{ML}}(\tau, \gamma)\right|_{\substack{\tau=\tau_{0} \\
\gamma=\gamma_{0}}}\right)^{2}\right\}}{\left(\mathrm{E}_{n(t)}\left\{\left.\frac{\partial^{2}}{\partial \tau \partial \tau} f_{\mathrm{ML}}(\tau, \gamma)\right|_{\substack{\tau=\tau_{0} \\
\gamma=\gamma_{0}}}\right\}\right)^{2}}\left[\frac{1}{\left(\mathrm{E}_{n(t)}\left\{\left.\frac{\partial^{2}}{\partial \tau \partial \tau} f_{\mathrm{ML}}(\tau, \gamma)\right|_{\substack{\tau=\tau_{0} \\
\gamma=\gamma_{0}}}\right\}\right)^{2}\left[\left(\left.\frac{\partial}{\partial \tau} f_{\mathrm{ML}}(\tau, \gamma)\right|_{\substack{\tau=\tau_{0} \\
\gamma=\gamma_{0}}}\right)^{2}\right.}\right. \\
& +\left(\gamma-\gamma_{0}\right)^{2}\left(\left.\frac{\partial^{2}}{\partial \tau \partial \gamma} f_{\mathrm{ML}}(\tau, \gamma)\right|_{\substack{\tau=\tau_{0} \\
\gamma=\gamma_{0}}}\right)^{2} \\
& \left.\left.+\left.\left.2\left(\gamma-\gamma_{0}\right) \frac{\partial}{\partial \tau} f_{\mathrm{ML}}(\tau, \gamma)\right|_{\substack{\tau=\tau_{0} \\
\gamma=\gamma_{0}}} \frac{\partial^{2}}{\partial \tau \partial \gamma} f_{\mathrm{ML}}(\tau, \gamma)\right|_{\substack{\tau=\tau_{0} \\
\gamma=\gamma_{0}}}\right\}\right] .
\end{aligned}
$$

The above expression of the MSE depends on the sign of the error between the true and the nominal values of the PLE. This dependence can be removed if we substitute the factor $\left.\frac{\partial^{2}}{\partial \tau \partial \gamma} f_{\mathrm{ML}}(\tau, \gamma)\right|_{\substack{\tau=\tau_{0} \\ \gamma=\gamma_{0}}}$ by its expectation, following a similar derivation in, e.g., [23], Equation (17-9.6) p. 642. Hence, we obtain an approximation of the MSE that is symmetric w.r.t. $\gamma_{0}$, namely

$$
\begin{aligned}
& \mathrm{E}_{n(t)}\left\{\left(\tau-\tau_{0}\right)^{2}\right\} \\
& \approx \mathrm{E}_{n(t)}\left\{\varepsilon_{\mathrm{TE}}^{2}\left(\tau_{0}, \gamma\right)\right\} \\
& \approx \frac{\mathrm{E}_{n(t)}\left\{\left(\left.\frac{\partial}{\partial \tau} f_{\mathrm{ML}}(\tau, \gamma)\right|_{\substack{\tau=\tau_{0} \\
\gamma=\gamma_{0}}}+\left(\gamma-\gamma_{0}\right) \mathrm{E}_{n(t)}\left\{\left.\frac{\partial^{2}}{\partial \tau \partial \gamma} f_{\mathrm{ML}}(\tau, \gamma)\right|_{\substack{\tau=\tau_{0} \\
\gamma=\gamma_{0}}}\right\}\right)^{2}\right\}}{\left(\mathrm{E}_{n(t)}\left\{\left.\frac{\partial^{2}}{\partial \tau \partial \tau} f_{\mathrm{ML}}(\tau, \gamma)\right|_{\substack{\tau=\tau_{0} \\
\gamma=\gamma_{0}}}\right\}\right)^{2}} .
\end{aligned}
$$

By substituting (24), (25), and (26) into (32), the MSE can be approximated by the closed formula (see Appendix 2 for details)

$$
\begin{aligned}
& \mathrm{E}_{n(t)}\left\{\varepsilon_{\mathrm{TE}}^{2}\left(\tau_{0}, \gamma\right)\right\} \\
& \approx \frac{\left(\gamma-\gamma_{0}\right)^{2} \frac{1}{4 \tau_{0}^{2}} \gamma_{0}^{2} a_{0}^{2} E_{\mathrm{s}}\left(\ln \left(\frac{d_{0}}{c \tau_{0}}\right)\right)^{2}+\frac{1}{2 \tau_{0}^{2}} \sigma_{\mathrm{n}}^{2} \gamma_{0}^{2}+8 \pi^{2} \bar{\beta}^{2} \sigma_{\mathrm{n}}^{2}}{E_{\mathrm{s}} a_{0}^{2}\left(\frac{1}{2 \tau_{0}^{2}} \gamma_{0}^{2}+8 \pi^{2} \bar{\beta}^{2}\right)^{2}} .
\end{aligned}
$$

Note that when $\gamma=\gamma_{0}$, the MSE of the approximate error reduces to

$$
\left.\mathrm{E}_{n(t)}\left\{\varepsilon_{\mathrm{TE}}^{2}\left(\tau_{0}, \gamma\right)\right\}\right|_{\gamma=\gamma_{0}}=\frac{1}{\frac{S}{N} a_{0}^{2}\left(8 \pi^{2} \bar{\beta}^{2}+\frac{\gamma_{0}^{2}}{2 \tau_{0}^{2}}\right)},
$$

which is identical to the expression in (9b).

\section{Performance in shadow fading environments}

In this section, we investigate the impact of the shadow fading on the analysis of Section 3. We also propose a Monte Carlo method for the numerical approximation of the MSE that avoids some of the approximations in the latter analysis.

\subsection{Analysis in presence of shadow fading}

The analytical approximations of the bias and the MSE of the ML estimator of the ToA are based on the assumption that the path gain can be modeled by Equation (7), which follows from neglecting the shadow fading term in Equation (3), i.e., taking $\psi_{\mathrm{dB}}=0$ in (3) or, equivalently, $\psi=1$ in Equation (6).

If shadow fading is explicitly taken into account, the path gain in Equation (6) becomes a random variable (because the factor $\psi$ is random with log-normal distribution). However, assuming that the channel noise $n(t)$ is independent of the shadow fading factor $\psi$, the analysis of Section 3.2 can still be carried out conditional on the random variable $\psi$.

To be specific, let

$$
a(\psi)=\sqrt{\frac{\kappa}{\psi}}\left(\frac{d_{0}}{c \tau}\right)^{\frac{1}{2} \gamma}
$$

be the (random) path gain associated to the pair of PLE and ToA values $\gamma$ and $\tau$, respectively. Correspondingly, let

$$
a_{0}(\psi)=\sqrt{\frac{\kappa}{\psi}}\left(\frac{d_{0}}{c \tau_{0}}\right)^{\frac{1}{2} \gamma_{0}}
$$

the gain associated to the true values $\gamma_{0}$ and $\tau_{0}$. In both cases, $\psi$ is a log-normal random variable, since $\psi_{\mathrm{dB}}=$ $10 \log _{10} \psi$ is Gaussian with zero mean and variance $\sigma_{\mathrm{dB}}^{2}$. If we repeat the analysis in Section 3.2 step by step, albeit 
conditional on the $\sigma$-algebra generated by the random variable $\psi$, we arrive at the approximations

$$
B\left(\tau_{0}, \gamma\right)=\left(\gamma-\gamma_{0}\right) \frac{\gamma_{0} \tau_{0} \ln \left(\frac{d_{0}}{c \tau_{0}}\right)}{\gamma_{0}^{2}+16 \pi^{2} \bar{\beta}^{2} \tau_{0}^{2}},
$$

for the bias of $\tau_{\mathrm{ML}}$ (see Equation (30)) and

$$
\begin{aligned}
& \varepsilon^{2}\left(\tau_{0}, \gamma, \psi\right) \\
& =\frac{\left(\gamma-\gamma_{0}\right)^{2} \frac{1}{4 \tau_{0}^{2}} \gamma_{0}^{2} a_{0}^{2}(\psi) E_{\mathrm{s}}\left(\ln \left(\frac{d_{0}}{c \tau_{0}}\right)\right)^{2}+\frac{1}{2 \tau_{0}^{2}} \sigma_{\mathrm{n}}^{2} \gamma_{0}^{2}+8 \pi^{2} \bar{\beta}^{2} \sigma_{\mathrm{n}}^{2}}{E_{\mathrm{s}} a_{0}^{2}(\psi)\left(\frac{1}{2 \tau_{0}^{2}} \gamma_{0}^{2}+8 \pi^{2} \bar{\beta}^{2}\right)^{2}}
\end{aligned}
$$

for the MSE of $\tau_{\mathrm{ML}}$. Note that the MSE in (38) is a random variable (while the MSE in Equation (33) is deterministic). Let us note that the approximate bias $B\left(\tau_{0}, \gamma\right)$ in (37) is independent of the path gain (and, therefore, of the shadow fading factor $\psi$ ) and, hence, deterministic. The approximate $\operatorname{MSE} \varepsilon^{2}\left(\tau_{0}, \gamma, \psi\right)$ of Equation (38), however, depends explicitly on $a_{0}(\psi)$; therefore, it is random. Let us look into its characterization.

When $\gamma=\gamma_{0}$, the MSE (as shown in Section 3.2) reduces to

$$
\left.\varepsilon^{2}\left(\tau_{0}, \gamma, \psi\right)\right|_{\gamma=\gamma_{0}}=\frac{1}{\frac{S}{N} a_{0}^{2}(\psi)\left(8 \pi^{2} \bar{\beta}^{2}+\frac{\gamma_{0}^{2}}{2 \tau_{0}^{2}}\right)},
$$

and it can be shown to be a log-normal random variable itself. In particular, if we write the approximate MSE when the PLE is perfectly known (i.e., $\gamma=\gamma_{0}$ ) in $\mathrm{dB}$,

$$
\left.\varepsilon^{2}\left(\tau_{0}, \gamma, \psi\right)_{\mathrm{dB}}\right|_{\gamma=\gamma_{0}}=10 \log _{10} \varepsilon^{2}\left(\tau_{0}, \gamma_{0}, \psi\right) \quad(d B),
$$

then it is straightforward to show that

$$
\begin{aligned}
\left.\varepsilon^{2}\left(\tau_{0}, \gamma, \psi\right)_{\mathrm{dB}}\right|_{\gamma=\gamma_{0}}= & \psi_{\mathrm{dB}}-20 \log _{10} a_{0}-10 \log _{10} \frac{S}{N} \\
& \times\left(8 \pi^{2} \bar{\beta}^{2}+\frac{\gamma_{0}^{2}}{2 \tau_{0}^{2}}\right),
\end{aligned}
$$

where $a_{0}=a_{0}(\psi=1)$ is the (deterministic) path gain in absence of shadow fading. Equation (41) reveals that the approximate MSE (in $\mathrm{dB}$ ) of the ML estimator of the ToA under a perfect knowledge of the PLE is a normal random variable with mean

$$
\begin{aligned}
\mathrm{E}_{\psi}\left\{\left.\varepsilon^{2}\left(\tau_{0}, \gamma, \psi\right)_{\mathrm{dB}}\right|_{\gamma=\gamma_{0}}\right\}= & -20 \log _{10} a_{0}-10 \log _{10} \frac{S}{N} \\
& \times\left(8 \pi^{2} \bar{\beta}^{2}+\frac{\gamma_{0}^{2}}{2 \tau_{0}^{2}}\right),
\end{aligned}
$$

and variance $\sigma_{\mathrm{dB}}^{2}$. Let us remark that this mean is in agreement with the approximation of Equation (34) in Section 3.2.

When $\gamma \neq \gamma_{0}$, the approximate $\operatorname{MSE} \varepsilon^{2}\left(\tau_{0}, \gamma, \psi\right)$ is given by the general expression of Equation (38). Let us rewrite it, for conciseness, as

$$
\varepsilon^{2}\left(\tau_{0}, \gamma, \psi\right)=C \psi+D,
$$

where

$$
\begin{aligned}
& C=\frac{1}{\frac{S}{N} a_{0}^{2}\left(\frac{1}{2 \tau_{0}^{2}} \gamma_{0}^{2}+8 \pi^{2} \bar{\beta}^{2}\right)}, \\
& D=\frac{\left(\gamma-\gamma_{0}\right)^{2} \frac{1}{4 \tau_{0}^{2}} \gamma_{0}^{2}\left(\ln \left(\frac{d_{0}}{c \tau_{0}}\right)\right)^{2}}{\left(\frac{1}{2 \tau_{0}^{2}} \gamma_{0}^{2}+8 \pi^{2} \bar{\beta}^{2}\right)^{2}},
\end{aligned}
$$

and we have used the fact that $a_{0}^{2}(\psi)=a_{0} / \psi$ (recall that $\left.a_{0}=a_{0}(\psi=1)\right)$. The random variable $\psi_{\mathrm{dB}}=10 \log _{10} \psi$ is normal with zero mean and variance $\sigma_{\mathrm{dB}}^{2}$. By a change of base in the logarithm, it is straightforward to show that the random variable $\ln \psi$ is also normal with zero mean and variance

$$
\operatorname{Var}(\ln \psi)=\frac{\sigma_{\mathrm{dB}}^{2}}{\left(10 \log _{10} e\right)^{2}} .
$$

This implies that the pdf of $\psi$ is [34]

$$
p_{\psi}(\psi)=\frac{10 \log _{10} e}{\psi \sigma_{\mathrm{dB}} \sqrt{2 \pi}} e^{-\frac{\left(10 \log _{10} e\right)^{2}}{2 \sigma_{\mathrm{dB}}^{2}}(\ln \psi)^{2}},
$$

and, in particular,

$$
\mathrm{E}\{\psi\}=e^{\frac{\sigma_{\mathrm{dB}}^{2}}{\left(10 \log _{10} e\right)^{2}}},
$$

$$
\operatorname{Var}\{\psi\}=e^{\frac{\sigma_{\mathrm{dB}}^{2}}{\left(10 \log _{10} e\right)^{2}}}\left(e^{\frac{\sigma_{\mathrm{dB}}^{2}}{\left(10 \log _{10} e\right)^{2}}}-1\right) .
$$

Since the approximate MSE, $\varepsilon^{2}\left(\tau_{0}, \gamma, \psi\right)$, is a linear transformation of $\psi$ (see Equation (43)), it is straightforward to calculate its mean

$$
\mu_{\varepsilon^{2}}=\mathrm{E}\left\{\varepsilon^{2}\left(\tau_{0}, \gamma, \psi\right)\right\}=D+C e^{\frac{\sigma_{\mathrm{dB}}^{2}}{\left(10 \log _{10} e\right)^{2}}},
$$

its variance

$\sigma_{\varepsilon^{2}}^{2}=\operatorname{Var}\left\{\varepsilon^{2}\left(\tau_{0}, \gamma, \psi\right)\right\}=C^{2} e^{\frac{\sigma_{\mathrm{dB}}^{2}}{\left(10 \log _{10} e\right)^{2}}}\left(e^{\frac{\sigma_{\mathrm{dB}}^{2}}{\left(10 \log _{10} e\right)^{2}}}-1\right)$,

and even its pdf

$$
p_{\mathrm{MSE}}\left(\varepsilon^{2}\right)=\frac{10 \log _{10} e}{\left(\varepsilon^{2}-D\right) \sigma_{\mathrm{dB}} \sqrt{2 \pi}} e^{-\frac{\left(10 \log _{10} e\right)^{2}}{2 \sigma_{\mathrm{dB}}^{2}}\left(\ln \left(\frac{\varepsilon^{2}-D}{C}\right)\right)^{2}} .
$$


Equations (50), (51), and (52) provide a complete statistical characterization of the approximate MSE of the ML estimator of the ToA in the presence of shadow fading.

\subsection{Monte Carlo approximation}

In the previous sections, we have introduced additional approximations beyond the linearization by Taylor series expansion (see Equations (23) and (32)) in order to attain closed form expressions for the bias and the MSE of the estimation error $\hat{\tau}_{\mathrm{ML}}(\gamma)-\tau_{0}$. One consequence of these approximations is that the formulas in (30) and (37) (for the bias), and (33) and (38) (for the MSE) may not properly represent the effect of the denominator in Equation (22), which can be relevant for the performance of the ML estimator $\hat{\tau}_{\mathrm{ML}}(\gamma)$ in the low SNR region. In this section, we address this limitation of the analysis by describing a simulation-based (Monte Carlo) method that enables us to obtain accurate numerical estimates of the bias and the MSE of the estimator $\hat{\tau}_{\mathrm{ML}}(\gamma)$ for a given nominal value $\gamma$ of the PLE in the presence of shadow fading. The technique only requires the ability to draw from a few Gaussian random variables, avoids some of the approximations in the analysis (namely that of Equation (23)), and has a computational cost well below that of the direct simulation of the transmission system.

We first seek an explicit expression of the estimation error

$$
\begin{aligned}
& \bar{\varepsilon}_{\mathrm{TE}}\left(\tau_{0}, \gamma, \psi\right) \\
& =-\frac{\left.\frac{\partial}{\partial \tau} f_{\mathrm{ML}}(\tau, \gamma)\right|_{\substack{\tau=\tau_{0} \\
\gamma=\gamma_{0}}}+\left(\gamma-\gamma_{0}\right) \mathrm{E}_{n(t)}\left\{\left.\frac{\partial^{2}}{\partial \tau \partial \gamma} f_{\mathrm{ML}}(\tau, \gamma)\right|_{\substack{\tau=\tau_{0} \\
\gamma=\gamma_{0}}}\right\}}{\left.\frac{\partial^{2}}{\partial \tau \partial \tau} f_{\mathrm{ML}}(\tau, \gamma)\right|_{\substack{\tau=\tau_{0} \\
\gamma=\gamma_{0}}}} .
\end{aligned}
$$

This error variable is similar to $\tilde{\varepsilon}_{\mathrm{TE}}\left(\tau_{0}, \gamma\right)$ in Equation (22) except for the expectation in the numerator, which is introduced to remove the effect in the MSE of the sign of the difference $\gamma-\gamma_{0}$ (as discussed in Section 3.2), and the explicit notation of the shadow fading factor $\psi$. Substituting (24), (29b), and

$$
\begin{aligned}
\left.\frac{\partial^{2}}{\partial \tau^{2}} f_{\mathrm{ML}}(\tau, \gamma)\right|_{\substack{\tau=\tau_{0} \\
\gamma=\gamma_{0}}}= & \frac{1}{2 \tau_{0}^{2}} \gamma_{0}^{2} E_{\mathrm{s}} a_{0}^{2}(\psi)+8 \pi^{2} \bar{\beta}^{2} E_{\mathrm{s}} a_{0}^{2}(\psi) \\
& -\frac{1}{\tau_{0}^{2}} \gamma_{0}\left(1+\frac{1}{2} \gamma_{0}\right) a_{0}(\psi) \rho_{\mathrm{ns}, 0} \\
& +\frac{1}{\tau_{0}} 2 \gamma_{0} a_{0}(\psi) \dot{\rho}_{\mathrm{ns}, 0}-2 a_{0}(\psi) \ddot{\rho}_{\mathrm{ns}, 0}
\end{aligned}
$$

(see Appendix 1) where $\ddot{\rho}_{\mathrm{ns}, 0}=\left.\frac{\partial^{2}}{\partial \tau^{2}} \rho_{\mathrm{ns}}\right|_{\substack{\tau=\tau_{0} \\ \gamma=\gamma_{0}}}$, into (53), we obtain the ToA estimation error

$$
\begin{aligned}
& \bar{\varepsilon}_{\mathrm{TE}}\left(\tau_{0}, \gamma\right) \\
& =-\frac{\frac{1}{\tau_{0}} \gamma_{0} \rho_{\mathrm{ns}, 0}-2 \dot{\rho}_{\mathrm{ns}, 0}-\left(\gamma-\gamma_{0}\right) \frac{1}{2 \tau_{0}} \gamma_{0} a_{0}(\psi) E_{\mathrm{s}} \ln \left(\frac{d_{0}}{c \tau_{0}}\right)}{\frac{1}{2 \tau_{0}^{2}} \gamma_{0}^{2} E_{\mathrm{s}} a_{0}(\psi)+8 \pi^{2} \bar{\beta}^{2} E_{\mathrm{s}} a_{0}(\psi)-\frac{1}{\tau_{0}^{2}} \gamma_{0}\left(1+\frac{1}{2} \gamma_{0}\right) \rho_{\mathrm{ns}, 0}+\frac{1}{\tau_{0}} 2 \gamma_{0} \dot{\rho}_{\mathrm{ns}, 0}-2 \ddot{\rho}_{\mathrm{ns}, 0}} .
\end{aligned}
$$

Note that $\bar{\varepsilon}_{\mathrm{TE}}\left(\tau_{0}, \gamma, \psi\right)$ is random because it depends on four random variables, $\rho_{\mathrm{ns}, 0}, \dot{\rho}_{\mathrm{ns}, 0}, \ddot{\rho}_{\mathrm{ns}, 0}$, and $a_{0}(\psi)$. The random path gain, $a_{0}(\psi)$, is related to the shadow fading factor $\psi$, which is log-normally distributed. Specifically, $\psi_{\mathrm{dB}}=10 \log _{10} \psi$ and $\psi_{\mathrm{dB}} \sim \mathcal{N}\left(0, \sigma_{\mathrm{dB}}^{2}\right)$.

The other three random variables $\rho_{\mathrm{ns}, 0}^{2}, \dot{\rho}_{\mathrm{ns}, 0}^{2}$, and $\ddot{\rho}_{\mathrm{ns}, 0}^{2}$, which, in turn, are related to the noise process $n(t)$ have a joint Gaussian distribution with zero mean vector and covariance matrix $\Sigma_{\rho}$, i.e.,

$$
\left[\begin{array}{c}
\rho_{\mathrm{ns}, 0} \\
\dot{\rho}_{\mathrm{ns}, 0} \\
\ddot{\rho}_{\mathrm{ns}, 0}
\end{array}\right] \sim \mathcal{N}\left(\mathbf{0}, \Sigma_{\rho}\right),
$$

where the covariance matrix $\boldsymbol{\Sigma}_{\rho}$ can be written as

$$
\boldsymbol{\Sigma}_{\rho}=\left[\begin{array}{ccc}
\mathrm{E}_{n(t)}\left\{\rho_{\mathrm{ns}, 0}^{2}\right\} & \mathrm{E}_{n(t)}\left\{\rho_{\mathrm{ns}, 0} \dot{\rho}_{\mathrm{ns}, 0}\right\} & \mathrm{E}_{n(t)}\left\{\rho_{\mathrm{ns}, 0} \ddot{\rho}_{\mathrm{ns}, 0}\right\} \\
\mathrm{E}_{n(t)}\left\{\rho_{\mathrm{ns}, 0} \dot{\rho}_{\mathrm{ns}, 0}\right\} & \mathrm{E}_{n(t)}\left\{\dot{\rho}_{\mathrm{ns}, 0}^{2}\right\} & \mathrm{E}_{n(t)}\left\{\dot{\rho}_{\mathrm{ns}, 0} \ddot{\rho}_{\mathrm{ns}, 0}\right\} \\
\mathrm{E}_{n(t)}\left\{\rho_{\mathrm{ns}, 0} \ddot{\rho}_{\mathrm{ns}, 0}\right\} & \mathrm{E}_{n(t)}\left\{\dot{\rho}_{\mathrm{ns}, 0} \ddot{\rho}_{\mathrm{ns}, 0}\right\} & \mathrm{E}_{n(t)}\left\{\ddot{\rho}_{\mathrm{ns}, 0}^{2}\right\}
\end{array}\right] .
$$

The expectation terms in (57), in turn, reduce to (see [35], Chapter 2)

$$
\begin{aligned}
& \mathrm{E}_{n(t)}\left\{\rho_{\mathrm{ns}, 0}^{2}\right\}=\frac{\sigma_{\mathrm{n}}^{2}}{2} \int_{0}^{T_{\mathrm{o}}} s^{2}(t) \mathrm{d} t=\frac{1}{2} E_{\mathrm{s}} \sigma_{\mathrm{n}}^{2}, \\
& \mathrm{E}_{n(t)}\left\{\dot{\rho}_{\mathrm{ns}, 0}^{2}\right\}=\frac{\sigma_{\mathrm{n}}^{2}}{2} \int_{0}^{T_{\mathrm{o}}}\left|\frac{\partial}{\partial \tau} s(t)\right|^{2} \mathrm{~d} t=2 \pi^{2} E_{\mathrm{s}} \bar{\beta}^{2} \sigma_{\mathrm{n}}^{2},
\end{aligned}
$$

$$
\begin{aligned}
\mathrm{E}_{n(t)}\left\{\ddot{\rho}_{\mathrm{ns}, 0}^{2}\right\} & =\frac{\sigma_{\mathrm{n}}^{2}}{2} \int_{0}^{T_{\mathrm{o}}}\left|\frac{\partial^{2}}{\partial \tau^{2}} s(t)\right|^{2} \mathrm{~d} t \\
\mathrm{E}_{n(t)}\left\{\rho_{\mathrm{ns}, 0} \dot{\rho}_{\mathrm{ns}, 0}\right\} & =\frac{\sigma_{\mathrm{n}}^{2}}{2} \int_{0}^{T_{\mathrm{o}}} s(t)\left(\frac{\partial}{\partial \tau} s(t)\right) \mathrm{d} t=0
\end{aligned}
$$

$$
\begin{aligned}
\mathrm{E}_{n(t)}\left\{\rho_{\mathrm{ns}, 0} \ddot{\rho}_{\mathrm{ns}, 0}\right\} & =\frac{\sigma_{\mathrm{n}}^{2}}{2} \int_{0}^{T_{\mathrm{o}}} s(t)\left(\frac{\partial^{2}}{\partial \tau^{2}} s(t)\right) \mathrm{d} t \\
& =-2 \pi^{2} E_{\mathrm{s}} \bar{\beta}^{2} \sigma_{\mathrm{n}}^{2} \\
\mathrm{E}_{n(t)}\left\{\dot{\rho}_{\mathrm{ns}, 0} \ddot{\rho}_{\mathrm{ns}, 0}\right\} & =\frac{\sigma_{\mathrm{n}}^{2}}{2} \int_{0}^{T_{\mathrm{o}}}\left(\frac{\partial}{\partial \tau} s(t)\right)\left(\frac{\partial^{2}}{\partial \tau^{2}} s(t)\right) \mathrm{d} t
\end{aligned}
$$


where the integrals in (58c) and (58f) do no not have a closed form but can be computed numerically.

In order to obtain accurate approximations of the statistics of the error $\bar{\varepsilon}_{\mathrm{TE}}\left(\tau_{0}, \gamma, \psi\right)$ we can resort to a numerical method based on Monte Carlo sampling. In particular, it is straightforward to draw an i.i.d. sample of size $N$ from the Gaussian random vector in (56), denoted as

$$
\left\{\rho_{\mathrm{ns}, 0}^{(i)}, \dot{\rho}_{\mathrm{ns}, 0}^{(i)}, \ddot{\rho}_{\mathrm{ns}, 0}^{(i)}, i=1, \ldots, N .\right\},
$$

as well as drawing $N$ i.i.d. samples of the Gaussian shadow fading variable $\psi_{\mathrm{dB}}$. If we let $\psi^{(i)}=10^{\frac{\psi_{\mathrm{dB}}^{(i)}}{10}}$, then it is straightforward to compute

$$
\left\{a_{0}^{(i)}, i=1, \ldots, N .\right\}
$$

where $a_{0}^{(i)}=a_{0}\left(\psi^{(i)}\right)$. Plugging the variates $\left(a_{0}^{(i)}, \rho_{\mathrm{ns}, 0}^{(i)}\right.$, $\dot{\rho}_{\mathrm{ns}, 0}^{(i)}$, and $\ddot{\rho}_{\mathrm{ns}, 0}^{(i)}$ ) into Equation (55) yields a size $N$ sample from the error distribution, denoted as

$$
\left\{\bar{\varepsilon}_{\mathrm{TE}}^{(i)}\left(\tau_{0}, \gamma\right), i=1, \ldots, N .\right\},
$$

and, given (61), the bias and the MSE of the ML estimate $\hat{\tau}_{\mathrm{ML}}\left(\tau_{0}, \gamma\right)$ can be approximated as

$$
\mathrm{E}_{n(t), \psi}\left\{\bar{\varepsilon}_{\mathrm{TE}}\left(\tau_{0}, \gamma, \psi\right)\right\} \approx \frac{1}{N} \sum_{i=1}^{N} \bar{\varepsilon}_{\mathrm{TE}}^{(i)}\left(\tau_{0}, \gamma\right)=\mu^{N}\left(\tau_{0}, \gamma\right),
$$

and

$\mathrm{E}_{n(t), \psi}\left\{\bar{\varepsilon}_{\mathrm{TE}}^{2}\left(\tau_{0}, \gamma, \psi\right)\right\} \approx \frac{1}{N} \sum_{i=1}^{N}\left(\bar{\varepsilon}_{\mathrm{TE}}^{(i)}\left(\tau_{0}, \gamma\right)\right)^{2}=\varepsilon^{2, N}\left(\tau_{0}, \gamma\right)$,

respectively. According to the Strong Law of Large Numbers, the estimates $\mu^{N}\left(\tau_{0}, \gamma\right)$ and $\varepsilon^{2, N}\left(\tau_{0}, \gamma\right)$ converge almost surely toward the true mean and the second order moment of $\bar{\varepsilon}_{\mathrm{TE}}\left(\tau_{0}, \gamma, \psi\right)$ ([36], Chapter 3). Note that this numerical approximation can be also applied assuming a deterministic path gain, i.e., in the absence of shadow fading effect, by simply setting $a_{0}=a_{0}(1)$ (i.e., $\psi=1$ ). In this case, the method requires drawing only from the random variables related to the noise $\left(\rho_{\mathrm{ns}, 0}^{(i)}, \dot{\rho}_{\mathrm{ns}, 0}^{(i)}\right.$, and $\left.\ddot{\rho}_{\mathrm{ns}, 0}^{(i)}\right)$.

Let us remark that the approximation procedure described in this section is semianalytical: it essentially relies on the error formula of (55), and the Monte Carlo simulation is only used as a numerical tool to integrate w.r.t. the random variables $a_{0}(\psi), \rho_{\mathrm{ns}, 0}$, and $\dot{\rho}_{\mathrm{ns}, 0} \ddot{\rho}_{\mathrm{ns}, 0}$. The simulations required are computationally 'cheap' compared to a full simulation of the communication system.

Finally, note also that a similar procedure to estimate the Friedlander's error derived in Section 3.1 is infeasible due to the fact that the error in Equation (19) depends on the random variables $\hat{\tau}_{\mathrm{ML}}, \hat{\gamma}_{\mathrm{ML}}, \rho\left(\hat{\tau}_{\mathrm{ML}}\right), \dot{\rho}\left(\hat{\tau}_{\mathrm{ML}}\right)$, and $\ddot{\rho}\left(\hat{\tau}_{\mathrm{ML}}\right)$, the probability density functions of which are unavailable. They are all related to the ML estimates of the ToA and the PLE ( $\hat{\tau}_{\mathrm{ML}}$ and $\left.\hat{\gamma}_{\mathrm{ML}}\right)$, which, in turn, depend also on the realization of the noise process $n(t)$.

\section{Numerical examples}

UWB signaling has been broadly studied as a promising candidate for accurate location estimation. In particular, UWB signaling is presented as an appropriate technology for positioning in indoor environments because it allows centimeter accuracy, as well as low-power and lowcost implementation of communication systems (see, e.g., $[4,37,38])$. For this reason, we have chosen to validate the analytical approximation results of Sections 3 and 4 by simulating the transmission of a second-derivative Gaussian pulse. This waveform is one of the most commonly used in classical impulse-radio UWB systems, and it can be expressed as [39])

$$
p(t)=\left(1-4 \pi\left(\frac{t}{\tau_{\mathrm{p}}}\right)^{2}\right) \mathrm{e}^{-2 \pi\left(\frac{t}{\tau_{\mathrm{p}}}\right)^{2}}, \quad t>0,
$$

where $\tau_{\mathrm{p}}$ is the pulse-shaping factor.

For the computer simulations in this section, we set $\tau_{\mathrm{p}}=$ $0.2877 \mathrm{~ns}$ to yield the pulse width $T_{\mathrm{p}}=0.7 \mathrm{~ns}$ and consider the transmitted signal $s(t)=p\left(t-\frac{1}{2} T_{\mathrm{p}}\right), \quad t>0$. The effective bandwidth, $\bar{\beta}$, and the effective absolute central frequency, $\bar{f}_{\mathrm{abs}}$, of $s(t)$ can be obtained analytically (see Appendices 3 and 4, respectively). To be specific,

$$
\begin{aligned}
\bar{\beta} & =\frac{1}{2 \pi} \sqrt{\frac{\int_{-\infty}^{\infty} \omega^{2}|S(\omega)|^{2} \mathrm{~d} \omega}{\int_{-\infty}^{\infty}|S(\omega)|^{2} \mathrm{~d} \omega}}=\frac{1}{\tau_{\mathrm{p}}} \sqrt{\frac{5}{2 \pi}}, \quad \text { and } \\
\bar{f}_{\text {abs }} & =\frac{1}{2 \pi} \frac{\int_{-\infty}^{\infty}|\omega||S(\omega)|^{2} \mathrm{~d} \omega}{\int_{-\infty}^{\infty}|S(\omega)|^{2} \mathrm{~d} \omega}=\frac{1}{\tau_{\mathrm{p}}} \frac{16}{3 \pi}
\end{aligned}
$$

where $\omega$ is the angular frequency, and $S(\omega)$ is the Fourier transform of $s(t)$. Note that $\bar{f}_{\text {abs }}$ is used here as an approximation of the central frequency in Equation (4), i.e., we assume $f_{0} \approx \bar{f}_{\text {abs }}$. By plugging the parameter values of (65a) and (65b) into Equations (30) and (33), we obtain an analytical characterization of the bias and the MSE, respectively, of the ML ToA estimator $\left(\hat{\tau}_{\mathrm{ML}}(\gamma)\right)$, conditional on the nominal PLE $\gamma$ for the second-derivative Gaussian pulse. If the shadow fading needs to be considered, (65a) and (65b) can be substituted into Equation (37) for the approximation of the bias and into Equations (50), (51), and (52) for the characterization of the random MSE. Similarly, we can substitute $\bar{\beta}$ and $f_{0} \approx \bar{f}_{\text {abs }}$ into Equation (55) in order to carry out a Monte Carlo evaluation of the statistics of the error $\bar{\varepsilon}_{\mathrm{TE}}\left(\tau_{0}, \gamma\right) \approx \hat{\tau}_{\mathrm{ML}}(\gamma)-\tau_{0}$.

In the remaining of this section, we numerically assess the validity of the approximation formulas that we have 
derived in Sections 3 and 4. In order to consider realistic scenarios, we select values for the PLE $\gamma_{0}$ and the variance of the shadow fading effect, $\sigma_{\mathrm{dB}}^{2}$, based on measurements in indoor environments [38].

\subsection{Path loss model}

Let us first consider the path loss model in the absence of shadow fading. Assuming a line-of-sight (LOS) wireless communication between a transmitter and a receiver in a residential environment, we use a typical value for the PLE as given in [38], e.g., $\gamma_{0}=1.7$. In order to validate the analytical characterization of the conditional ML estimator $\left(\hat{\tau}_{\mathrm{ML}}(\gamma)\right)$ obtained in Section 3, we compare the approximate bias and RMSEs computed by means of the formulas in both sections with the results obtained from the direct simulation of the transmission system. In particular, we consider the following methods to evaluate both the bias and the RMSE of the estimator:

- Direct simulation of the transmission system with either perfect knowledge of the PLE ( $\gamma=\gamma_{0}$, i.e., $\left.\delta_{\gamma}=0\right)$ or imperfect PLE $\left(\gamma \neq \gamma_{0}\right.$, i.e., $\left.\delta_{\gamma} \neq 0\right)$. We run $N_{\mathrm{R}}=6,000$ independent simulations and compute the conditional ML estimator ${ }^{\mathrm{b}} \hat{\tau}_{\mathrm{ML}}(\gamma)$ and the errors $\hat{\tau}_{\mathrm{ML}}(\gamma)-\tau_{0}$ and $\left(\hat{\tau}_{\mathrm{ML}}(\gamma)-\tau_{0}\right)^{2}$ in each case. The errors are then averaged and displayed.

- The approximate formula of Section 2 for the MSE of the ML estimator, which can only be applied with a perfect PLE $\left(\gamma=\gamma_{0}\right.$, i.e., $\left.\delta_{\gamma}=0\right)$.

- The approximate formula of Equations (30) and (33) for the bias and the RMSE, respectively, of the ML estimator. Such approximation can be used with the imperfect PLE $\left(\gamma \neq \gamma_{0}\right)$.

- With an imperfect PLE $\left(\delta_{\gamma} \neq 0\right)$, we can also compute the (approximate) expected bias and RMSE via the Monte Carlo approach in Section 4.2, using a population $N=6,000$ samples. Note that in this case, we consider the deterministic path gain given in (7) (no shadow fading); therefore, it is only necessary to draw from the Gaussian variables $\rho_{\mathrm{ns}, 0}^{(i)}, \dot{\rho}_{\mathrm{ns}, 0}^{(i)}$, and $\ddot{\rho}_{\mathrm{ns}, 0}^{(i)}$.

For a better display, the errors (bias and RMSE) are shown in terms of the transmitter-receiver distance $d=$ $c \tau$, where $c$ is the speed of the light. For an arbitrary estimate $\hat{\tau}$, the bias and the RMSE of the corresponding distance estimate $\hat{d}=c \hat{\tau}$ are proportional (with constant $c$ ) to the bias and the RMSE of $\hat{\tau}$.

Figure 2 shows the bias of the estimated distance as a function of the received SNR, defined as SNR = $10 \log _{10}\left(\frac{a^{2} E_{\mathrm{s}}}{\sigma_{\mathrm{n}}^{2}}\right)(\mathrm{dB})$ (with $a$ as given in Equation (7)). The upper plot shows that the analytical expression properly captures the results from the direct simulation for medium to high $\mathrm{SNR}$ values $(\mathrm{SNR} \geq 20 \mathrm{~dB}$ ). The lower plot is a magnification of the vertical axis (notice the range of $15 \times 10^{-5} \mathrm{~m}$ ) that shows the good fit between the analytical formula for the bias and the results of simulations, both for positive and negative deviations of the nominal PLE. Note also that the bias of the estimated distance is positive when the PLE error is negative and vice-versa, as predicted by Equation (30). The physical interpretation of this result comes from the fact that the path gain $a$ decreases both with the ToA $\tau$ and with the PLE $\gamma$, as explicitly shown in Equation (7).

Figure 3 shows the bias of the estimated distance as a function of PLE error given by $\delta_{\gamma}=\gamma-\gamma_{0}$. The upper plot shows that the analytical bias is a good approximation of the simulation results only for PLE errors greater than $\delta_{\gamma}=-0.5$, due to the asymmetric behavior of the conditional ML estimator w.r.t. the sign of $\delta_{\gamma}$ (this is in agreement with the plot of Figure 1 , which shows that $f_{\mathrm{ML}}$ is not symmetric around the true PLE value). From the lower plot (a magnification of the vertical axis with a range of $8 \times 10^{-5} \mathrm{~m}$ ), we can observe that the theoretical analysis is more accurate for small values of the PLE error $\delta_{\gamma}$, i.e., for $\delta_{\gamma}$ approximately between -0.5 and 0.2 . This is as expected, because the Taylor series expansion for two variables is accurate only in the neighborhood of the true values of both variables. This figure also shows the performance of the ML estimator under a perfect knowledge of the PLE, which is unbiased and serves as a practical performance reference.

In Figure 4, we show the RMSE of the estimated distance as a function of the received SNR. Again, the analytical approximations (of the RMSE) turn out accurate for medium to high SNRs. The Monte Carlo approximations (for both perfect and imperfect PLE) of the RMSE provide better results than the analytical approximations (in Equations (9b) for the perfect PLE and (33) for the imperfect PLE) for the low SNR region, while for medium to high SNRs, both the analytical and the Monte Carlo approximations yield similar results.

Finally, Figure 5 shows the RMSE of the estimated distance as a function of the PLE error for several values of the received SNR. As before, we can observe that the analysis is more accurate in the vicinity of the true value of the PLE $\left(\delta_{\gamma}=0\right)$. Note also that the approximation works properly for SNR above $20 \mathrm{~dB}$ and $\delta_{\gamma}>-0.4$.

\subsection{Shadow fading environment}

In this section, we validate the analytical and Monte Carlo approximations derived in Section 4 for a combined path loss and shadow fading model. To this end, we consider the ToA estimation in a residential environment in two different scenarios:

1. An LOS exists between the transmitter and the receiver of the wireless communication system, 

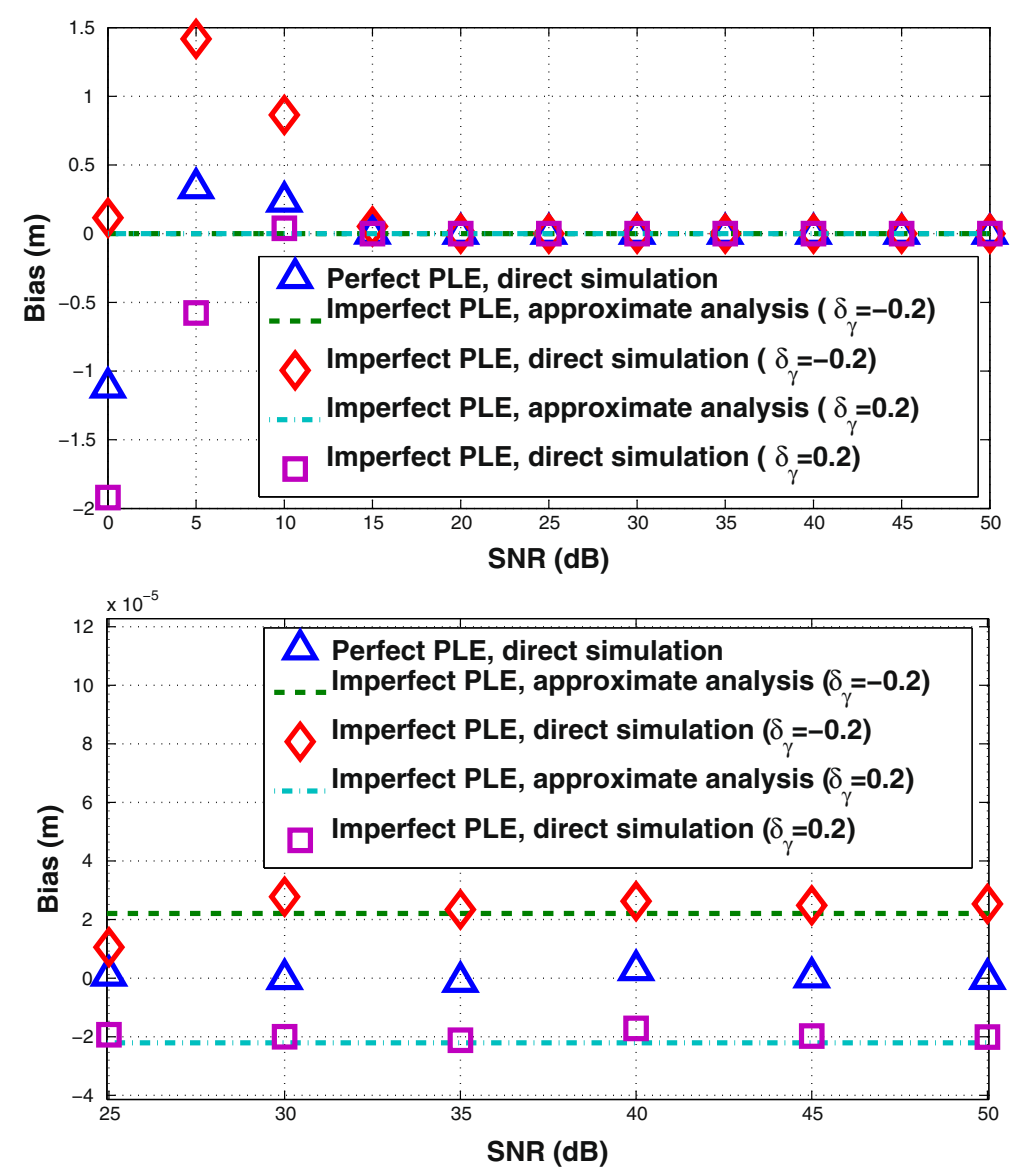

Figure 2 The bias of the estimated distance as a function of the received SNR. Top: Bias of the ML distance estimate as a function of the SNR with $\gamma_{0}=1.7, \delta_{\gamma}= \pm 0.2, c \tau_{0}=4 \mathrm{~m}, \bar{\beta}=3.1007 \times 10^{9} \mathrm{~Hz}$, sampling time $=0.01 \mathrm{ps}$, and $N_{\mathrm{R}}=6,000$ independent runs. Bottom: A magnification of the upper plot.

where the typical values for the PLE and the variance of the shadow fading are, e.g., $\gamma_{0}=1.7$ and $\sigma_{\mathrm{dB}}^{2}=1.6$, respectively [38].

2. There is no LOS (NLOS) between the transmitter and the receiver of the wireless communication system. The typical values for the PLE and the variance of the shadow fading are, e.g., $\gamma_{0}=3.5$ and $\sigma_{\mathrm{dB}}^{2}=7.29$ [38].

In order to validate the analytical and the numerical characterizations of the conditional ML estimator, $\hat{\tau}_{\mathrm{ML}}(\gamma)$, obtained in Section 4, we compare the approximate bias and RMSEs with the results obtained from the direct simulation of the transmission system. Similar to Section 5.1, we consider the following methods to evaluate the bias and the RMSE of the ML estimator $\hat{\tau}_{\mathrm{ML}}(\gamma)$ :

- Direct simulation of the transmission system in the both LOS and NLOS communication, with either perfect $\left(\gamma=\gamma_{0}\right)$ or imperfect knowledge of imperfect PLE $\left(\gamma \neq \gamma_{0}\right)$. We run $N_{R}=6,000$ independent simulations and compute the ML estimator $\hat{\tau}_{\mathrm{ML}}(\gamma)$ and the errors $\hat{\tau}_{\mathrm{ML}}(\gamma)-\tau_{0}$ and $\left(\hat{\tau}_{\mathrm{ML}}(\gamma)-\tau_{0}\right)^{2}$ in each one of them. The errors are then averaged and displayed.

- With an imperfect PLE $\left(\gamma \neq \gamma_{0}\right)$, we can approximate the RMSE by using the approximate analysis of the random MSE $\varepsilon^{2}$ obtained in Section 4.2. In particular, we can approximate the RMSE of the ML estimator $\hat{\tau}_{\mathrm{ML}}(\gamma)$ by the square root of the mean $\mu_{\varepsilon^{2}}$ in Equation (50).

- With an imperfect PLE $\left(\gamma \neq \gamma_{0}\right)$, we can approximate the expected bias and RMSE via the Monte Carlo approach in Section 4.2, using $N=6,000$ samples of the random error $\bar{\varepsilon}_{T E}\left(\tau_{0}, \gamma\right)$ in Equation (55). Note that in this section, we are considering the shadow fading effect; thus, the path gain is a random variable related to the random shadow fading term $\psi_{\mathrm{dB}}$, as shown in Equation (6). Therefore, the Monte Carlo approach requires to draw samples from four Gaussian variables $\rho_{\mathrm{ns}, 0}^{(i)}$, $\dot{\rho}_{\mathrm{ns}, 0}^{(i)}, \ddot{\rho}_{\mathrm{ns}, 0}^{(i)}$, and $\psi_{\mathrm{dB}}^{(i)}, i=1, \ldots, N$. 

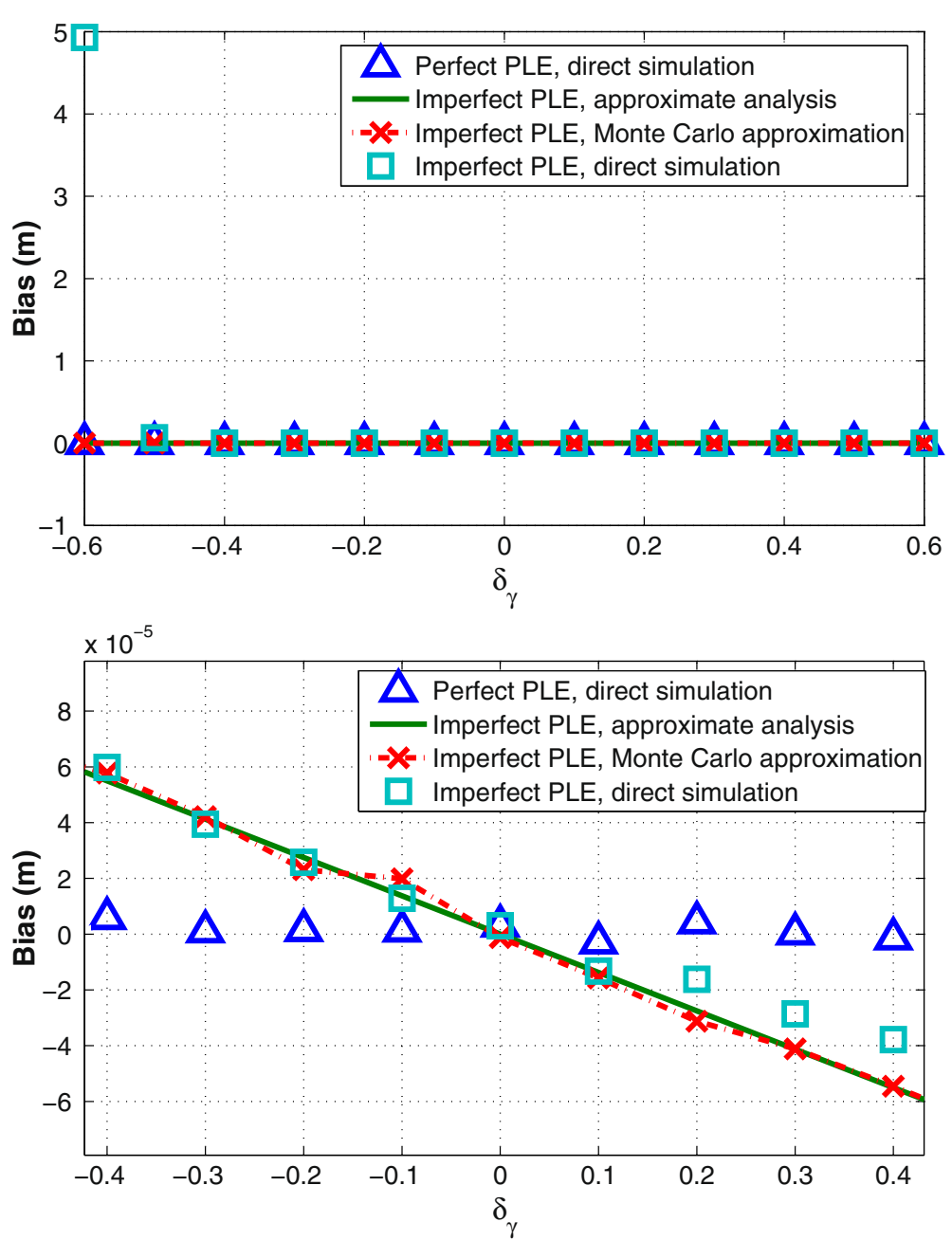

Figure 3 Bias of the estimated distance as a function of PLE error. Top: Bias of the ML distance estimate as a function of the PLE error $\delta_{\gamma}$, with $\gamma_{0}=1.7, \mathrm{SNR}=30 \mathrm{~dB}, c \tau_{0}=4 \mathrm{~m}, \bar{\beta}=3.1007 \times 10^{9} \mathrm{~Hz}$, sampling time $=0.01 \mathrm{ps}$, and $N_{\mathrm{R}}=6,000$ independent runs. Bottom: A magnification of the upper plot.

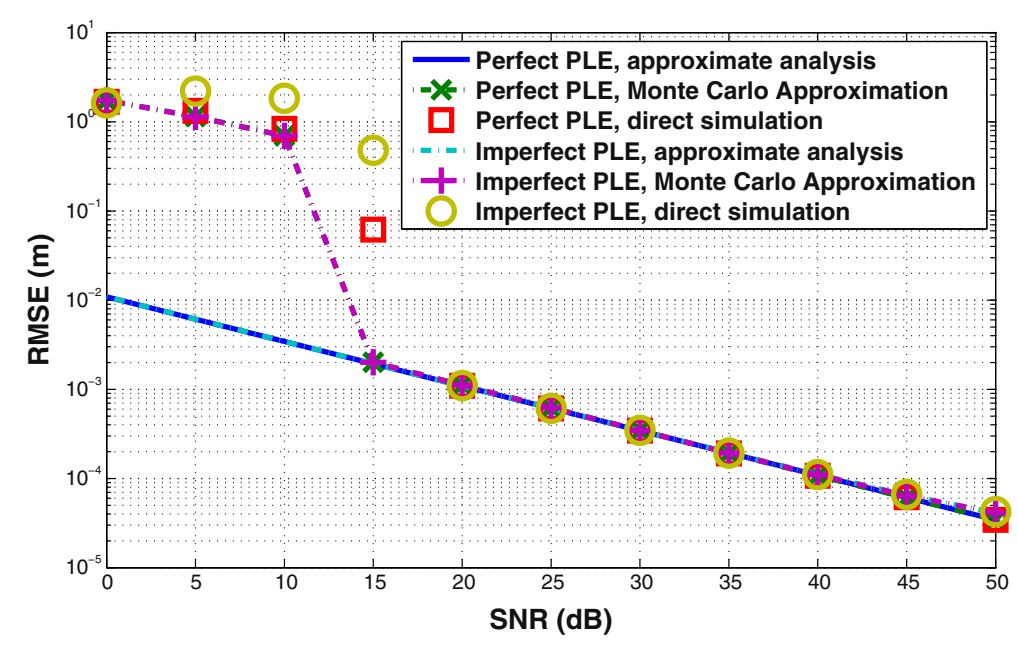

Figure 4 RMSE of the estimated distance as a function of the received SNR. RMSE of the ML distance estimate as a function of the SNR with $\gamma_{0}=1.7, \delta_{\gamma}=-0.2, c \tau_{0}=4 \mathrm{~m}, \bar{\beta}=3.1007 \times 10^{9} \mathrm{~Hz}$, sampling time $=0.01 \mathrm{ps}$, and $N_{R}=6,000$ independent runs. 


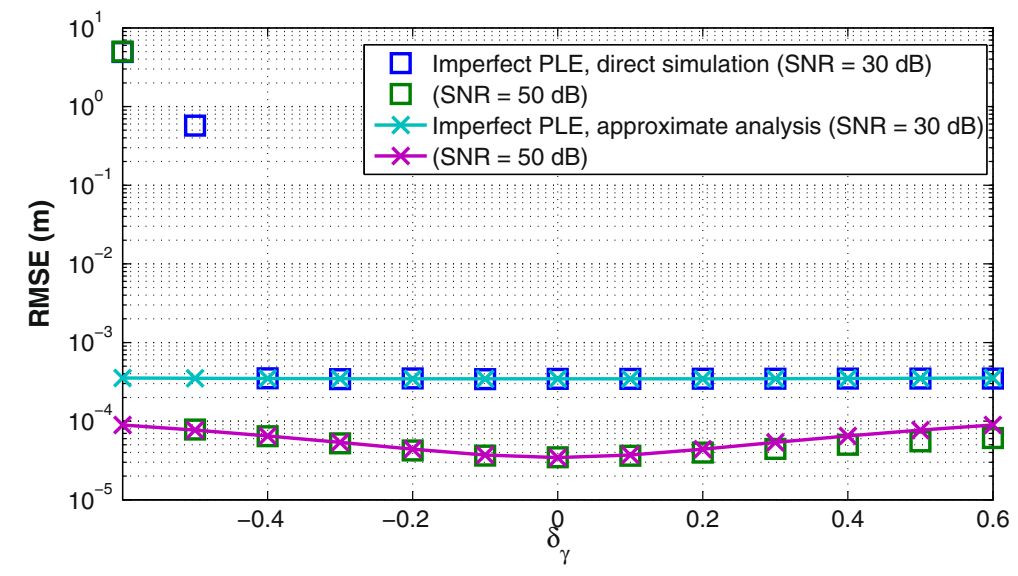

Figure 5 RMSE of the estimated distance as a function of the PLE error. RMSE of the ML distance estimate as a function of the PLE error $\delta_{\gamma}$ for several values of the SNR, with $\gamma_{0}=1.7, c \tau_{0}=4 \mathrm{~m}, \bar{\beta}=3.1007 \times 10^{9} \mathrm{~Hz}$, sampling time $=0.01 \mathrm{ps}$, and $N_{\mathrm{R}}=6,000$ independent runs.

The errors (bias and RMSE) are shown in terms of the transmitter-receiver distance $d=c \tau$ for a better display. Figure 6 shows the bias and the RMSE of the estimated distance as a function of received SNR, assuming LOS between the transmitter and the receiver of the communication system. The upper plot shows that the numerical approximation of the bias properly captures the results of the direct simulation for high SNR values and for both positive and negative deviations of the nominal PLE (notice that the range of the vertical axis is only $10^{-4} \mathrm{~m}$ ). The lower plot shows the Monte Carlo method approximation of the RMSE for a range of SNR values. This figure also shows that a negative deviation of the PLE leads to a greater RMSE than a positive error. This means that the ML estimator of the ToA for a negative PLE mismatch $\left(\delta_{\gamma}=\gamma-\gamma_{0}<0\right)$ is more sensitive to the effects of noise and shadow fading.

In Figure 7, we show the RMSE of the estimated distance as a function of the received SNR, assuming NLOS between the transmitter and the receiver (hence, with larger values of $\gamma_{0}$ and $\sigma_{\mathrm{dB}}^{2}$ ) and $\delta_{\gamma}=\gamma-\gamma_{0}=0.2$. This figure shows that the analytical characterization of the random MSE provided in Section 4.1 is accurate only in the high SNR region. This figure also shows that the Monte Carlo approximation of the RMSE is accurate in the low and the high SNR regions. In the medium SNR region, we observe a larger mismatch.

\subsection{Impact of the approximations}

In Sections 3 and 4, we have introduced additional approximations, beyond the linearization by Taylor series expansion, in order to attain analytical expressions for the bias and the MSE of the estimator $\hat{\tau}_{\mathrm{ML}}(\gamma)$. The goal of this section is to summarize and point out the impact of such approximations.
In Section 3, we have taken the expectation w.r.t. the noise process $n(t)$ in the denominator of Equation (22). One consequence of this approximation is that the formulas in (30) and (37) (for the bias), and (33) and (38) (for the MSE) do not properly capture the effect of the denominator in Equation (22), which is relevant for the performance of the ML estimator $\hat{\tau}_{M L}(\gamma)$ in the low SNR region. Although the impact of this approximation cannot be analyzed theoretically, the numerical results in Figure 4 show that the mismatch between the proposed analytical approximation of the RMSE and the RMSE obtained by direct simulation of the communication system is larger in the low SNR region. This artifact is considerably mitigated when the RMSE is approximated with the Monte Carlo method of Section 4.2. Indeed, it can be seen in Figures 4, 6, and 7 (the latter for a shadow fading scenario) that the Monte Carlo estimates of the RMSE are usable in the whole SNR range (although still better for high values, SNR $>20 \mathrm{~dB}$ ). Note that the Monte Carlo procedure of Section 4.2 requires to draw only from a few simple Gaussian distributions, which is computationally much cheaper than simulating the complete transmission system.

The Taylor series expansion leads to an approximation of the MSE that depends on the sign of the difference $\delta_{\gamma}=\gamma-\gamma_{0}$ between the true and the nominal values of the PLE, as shown in Equation(31). We have followed [23, equation (17-9.6), p. 642] in order to remove this dependence, leading to Equation (32). As a consequence, the analytical and the Monte Carlo approximations of the RMSE proposed in the paper are independent of the sign of $\delta_{\gamma}$. While this approximation is correct for small $\delta_{\gamma}$, the simulations (see Figures 2, 3, 5, and 6) show that the estimator is more sensitive to negative errors in the PLE. 

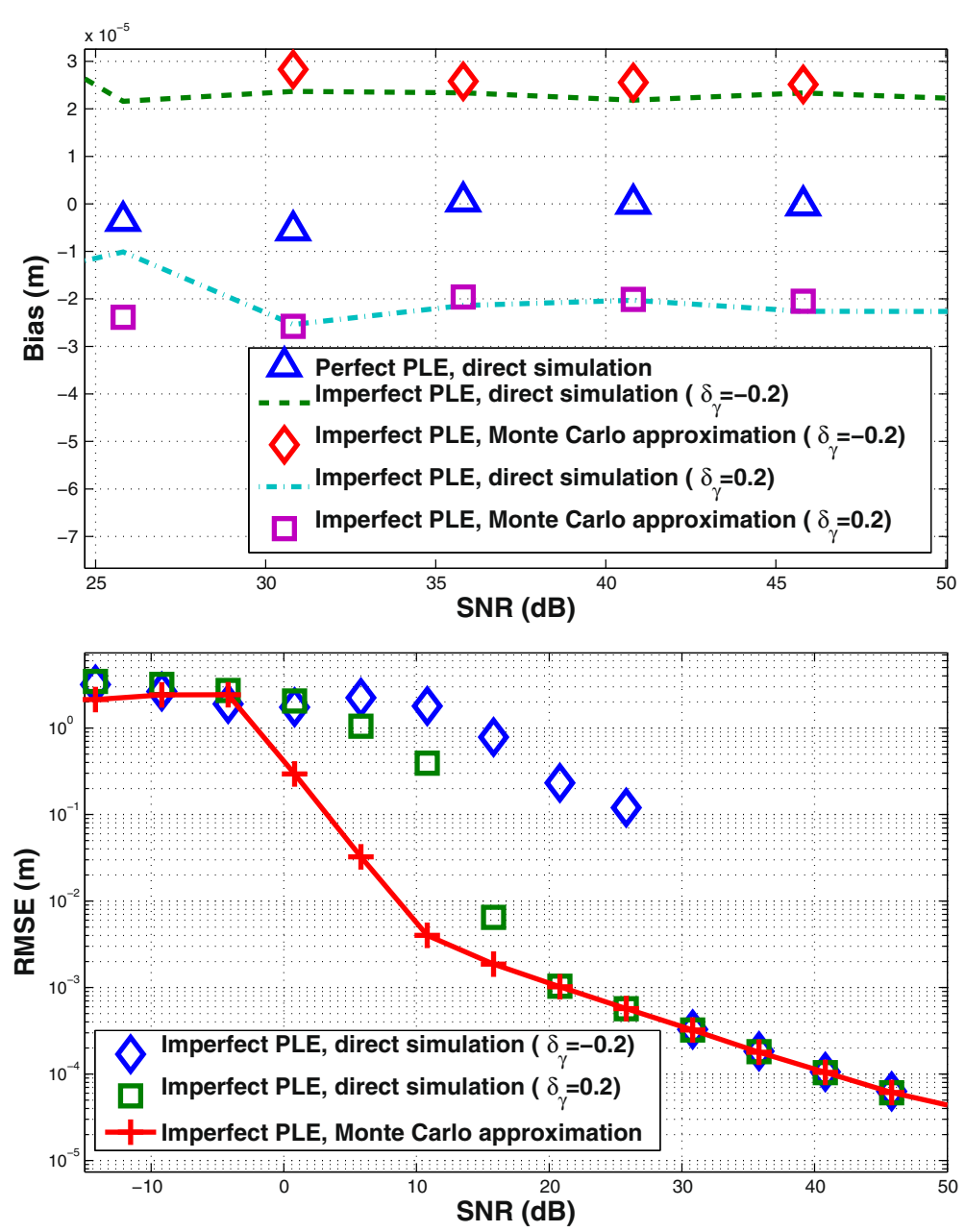

Figure 6 Bias (top) and RMSE (bottom) of the estimated distance as a function of the SNR. LOS transmission with $\gamma_{0}=1.7, \delta_{\gamma}= \pm 0.2$, $\sigma_{\mathrm{dB}}^{2}=1.6, c \tau_{0}=4 \mathrm{~m}, \bar{\beta}=3.1007 \times 10^{9} \mathrm{~Hz}$, sampling time $=0.01 \mathrm{ps}$, and $N_{\mathrm{R}}=6,000$ independent runs. Note that the Monte Carlo approximation of the RMSE is independent of the sign of $\delta_{\gamma}$.

\section{Conclusions}

We have analyzed the performance of the maximum likelihood of a signal time-of-arrival when the path loss exponent of the communication link is not perfectly known. In the first approach, we have modeled the signal received amplitude as a deterministic function of the PLE and the transmitter-to-receiver distance. Within this setup, we have applied a Taylor series expansion, together with other approximations needed for mathematical tractability, in order to obtain closed-form expressions for the bias and the RMSE of the ML ToA estimator. In the second stage, we have extended our analysis to cope with shadow fading effects. In such case, the analytical approximation of the estimator MSE takes the form of a random variable, the mean, variance, and probability density function of which are derived and given in closed form. Additionally, we have introduced a simple
Monte Carlo method for the numerical computation of the errors, which removes some of the approximations in the analysis, can be applied both with and without shadow fading, and presents a computational load much smaller than that of the direct simulation of the transmission.

We have carried out extensive computer simulations to assess the validity of the proposed approximation tools. For the evaluation, we have simulated the transmission of a second derivative Gaussian pulse, a waveform commonly used in UWB systems, where the ToA estimation is of great importance for positioning applications. Our simulations show that the analytical approximations, both with and without shadow fading, are very accurate in the medium and high SNR regions. The Monte Carlo technique is equally accurate with mid and high SNRs, while it also yields usable approximations of the bias and RMSE 


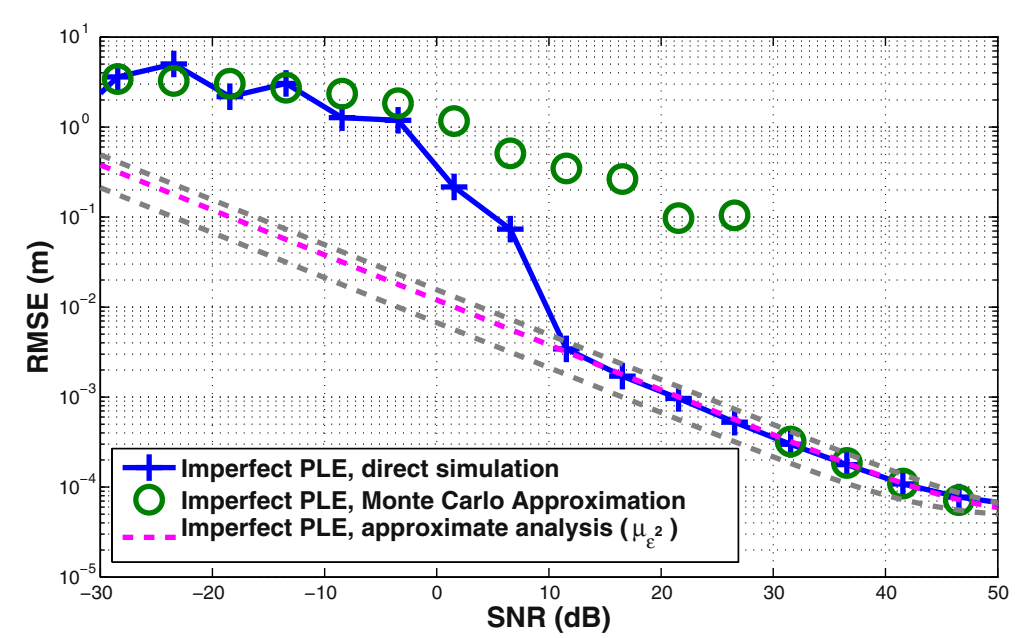

Figure 7 RMSE of the ML distance estimate as a function of the SNR. NLOS transmission with $\gamma_{0}=3.5, \delta_{\gamma}=0.2, \sigma_{\mathrm{dB}}^{2}=7.29, c \tau_{0}=4 \mathrm{~m}$, $\bar{\beta}=3.1007 \times 10^{9} \mathrm{~Hz}$, sampling time $=0.01 \mathrm{ps}$, and $N_{R}=6,000$ independent runs. Recall that we have modeled the MSE as a random variable with mean $\mu_{\varepsilon^{2}}$ (Equation (50)) and variance $\sigma_{\varepsilon^{2}}^{2}$ (Equation (51)). The analytical approximation of the RMSE of the ML ToA estimation is $\sqrt{\mu_{\varepsilon^{2}}}$, and we also show $\sqrt{\mu_{\varepsilon^{2}} \pm \sigma_{\varepsilon^{2}}}$ in the plot.

in the lower SNR region. The computer experiments also show that the ML ToA estimator is more sensitive to the underestimation of the PLE than to its overestimation (this result is consistent with the numerical study of [6]).

\section{Endnotes}

a Actually, the negative of the log-likelihood.

b We have solved the optimization problem of Equation (12) numerically in order to compute $\hat{\tau}_{\mathrm{ML}}(\gamma)$. In particular, we have generated a regular grid of candidate values of $\tau$ (with separation of $10^{-2} \mathrm{ps}$ between adjacent points of the grid), evaluated the likelihood $f_{\mathrm{ML}}$ for every candidate, and then selected the best one. It is not the goal of this paper to propose a practical means for the calculation of $\hat{\tau}_{\mathrm{ML}}(\gamma)$, but simply to assess its theoretical performance. In a practical receiver, an adaptive algorithm (similar to a timing error detector [40]) could be used to compute $\hat{\tau}_{\mathrm{ML}}(\gamma)$.

\section{Appendices}

\section{Algebraic derivations}

Appendix 1: derivatives of the likelihood function $f_{\mathrm{ML}}(\tau, \gamma)$ The objective of this appendix is to obtain closed expressions for the derivatives of the likelihood function $f_{\mathrm{ML}}(\tau, \gamma)$, which are needed in Section 3.2. The partial derivatives of the likelihood function $f_{\mathrm{ML}}(\tau, \gamma)$ w.r.t. $\tau, \tau^{2}$, and $\gamma$ are given by

$$
\frac{\partial}{\partial \tau} f_{\mathrm{ML}}(\tau, \gamma)=-\frac{1}{\tau} \gamma\left(E_{\mathrm{s}} a-\rho(\tau)\right) a-2 a \frac{\partial}{\partial \tau} \rho(\tau),
$$

$$
\begin{aligned}
\frac{\partial^{2}}{\partial \tau^{2}} f_{\mathrm{ML}}(\tau, \gamma)= & \frac{1}{\tau^{2}} \gamma(1+\gamma) E_{\mathrm{s}} a^{2}-\frac{1}{\tau^{2}} \gamma\left(1+\frac{1}{2} \gamma\right) \\
& \times a \rho(\tau)+\frac{1}{\tau} 2 \gamma a \frac{\partial}{\partial \tau} \rho(\tau)-2 a \frac{\partial^{2}}{\partial \tau^{2}} \rho(\tau),
\end{aligned}
$$

$$
\begin{aligned}
\frac{\partial^{2}}{\partial \gamma \partial \tau} f_{\mathrm{ML}}(\tau, \gamma)= & \frac{1}{\tau} a\left(\rho(\tau)-E_{\mathrm{s}} a\right)+\frac{1}{\tau} \gamma \ln \left(\frac{d_{0}}{c \tau}\right) \\
& \times a\left(\frac{1}{2} \rho(\tau)-E_{\mathrm{S}} a\right)-\ln \left(\frac{d_{0}}{c \tau}\right) \\
& \times a \frac{\partial}{\partial \tau} \rho(\tau),
\end{aligned}
$$

respectively, where the correlation $\rho(\tau)$ can be written as

$$
\rho(\tau)=\int_{0}^{T_{\mathrm{o}}} \Re\left(r(t) s^{*}(t-\tau)\right) \mathrm{d} t=a_{0} \rho_{\mathrm{ss}}(\tau)+\rho_{\mathrm{ns}}(\tau) .
$$

The functions $\rho_{\mathrm{SS}}(\tau)$ and $\rho_{\mathrm{ns}}(\tau)$, given by Equations (17) and (18), evaluated at $\tau=\tau_{0}$ yield

$$
\begin{aligned}
& \rho_{\mathrm{ss}}\left(\tau_{0}\right)=\int_{0}^{T_{0}}|s(t)|^{2} \mathrm{~d} t=E_{s}, \quad \text { and } \\
& \rho_{\mathrm{ns}}\left(\tau_{0}\right)=\rho_{\mathrm{ns}, 0} .
\end{aligned}
$$

Hence, the expression of $\rho(\tau)$ for $\tau=\tau_{0}$ is given by

$$
\rho\left(\tau_{0}\right)=a_{0} E_{s}+\rho_{\mathrm{ns}, 0} .
$$


Moreover, the derivative of $\frac{\partial}{\partial \tau} \rho(\tau)$ evaluated at $\tau=\tau_{0}$ can be written as

$$
\left.\frac{\partial}{\partial \tau} \rho(\tau)\right|_{\tau=\tau_{0}}=\left.a_{0} \frac{\partial}{\partial \tau} \rho_{\mathrm{ss}}(\tau)\right|_{\tau=\tau_{0}}+\left.\frac{\partial}{\partial \tau} \rho_{\mathrm{ns}}(\tau)\right|_{\tau=\tau_{0}},
$$

where $\left.\frac{\partial}{\partial \tau} \rho_{\text {ss }}(\tau)\right|_{\tau=\tau_{0}}$ can be obtained as

$$
\left.\frac{\partial}{\partial \tau} \rho_{\mathrm{SS}}\right|_{\tau=\tau_{0}}=\left.\int_{0}^{T_{\mathrm{o}}} \Re\left(s\left(t-\tau_{0}\right) \frac{\partial}{\partial \tau} s^{*}(t-\tau)\right) \mathrm{d} t\right|_{\tau=\tau_{0}} .
$$

Applying the change of variable $t^{\prime}=t-\tau$ in (73), we can write

$$
\begin{aligned}
\left.\frac{\partial}{\partial \tau} \rho_{\mathrm{SS}}\right|_{\tau=\tau_{0}} & =\left.\int_{-\tau}^{T_{\mathrm{o}}-\tau} \mathfrak{R}\left(s\left(t^{\prime}-\left(\tau_{0}-\tau\right)\right) \frac{\partial}{\partial t^{\prime}} s^{*}\left(t^{\prime}\right)\right) \mathrm{d} t^{\prime}\right|_{\tau=\tau_{0}} \\
& =\int_{-\tau_{0}}^{T_{\mathrm{o}}-\tau_{0}} \mathfrak{R}\left(s\left(t^{\prime}\right) \frac{\partial}{\partial t^{\prime}} s^{*}\left(t^{\prime}\right)\right) \mathrm{d} t^{\prime} \\
& =\left.\frac{1}{2}\left|s\left(t^{\prime}\right)\right|^{2}\right|_{t^{\prime}=-\tau_{0}} ^{t^{\prime}=T_{0}-\tau_{0},},
\end{aligned}
$$

in which the change of variable $t=t^{\prime}+\tau_{0}$ yields

$$
\left.\frac{\partial}{\partial \tau} \rho_{\mathrm{ss}}\right|_{\tau=\tau_{0}}=\left.\frac{1}{2}|s(t)|^{2}\right|_{t=0} ^{t=T_{\mathrm{o}}}=0
$$

Then, by defining $\dot{\rho}_{\mathrm{ns}, 0}=\left.\frac{\partial}{\partial \tau} \rho_{\mathrm{ns}}(\tau)\right|_{\tau=\tau_{0}},(72)$ reduces to

$$
\left.\frac{\partial}{\partial \tau} \rho(\tau)\right|_{\tau=\tau_{0}}=\dot{\rho}_{\mathrm{ns}, 0}
$$

Substituting (71) and (76) into (66) and (68), we obtain the first and the second derivatives, $\frac{\partial}{\partial \tau} f_{\mathrm{ML}}(\tau, \gamma)$ and $\frac{\partial^{2}}{\partial \gamma \partial \tau} f_{\mathrm{ML}}(\tau, \gamma)$, evaluated at $\tau_{0}$ and $\gamma_{0}$ as

$$
\left.\frac{\partial}{\partial \tau} f_{\mathrm{ML}}(\tau, \gamma)\right|_{\substack{\tau=\tau_{0} \\ \gamma=\gamma_{0}}}=\frac{1}{\tau_{0}} \gamma_{0} a_{0} \rho_{\mathrm{ns}, 0}-2 a_{0} \dot{\rho}_{\mathrm{ns}, 0}, \quad \text { and }
$$

$$
\begin{aligned}
\left.\frac{\partial^{2}}{\partial \gamma \partial \tau} f_{\mathrm{ML}}(\tau, \gamma)\right|_{\substack{\tau=\tau_{0} \\
\gamma=\gamma_{0}}}= & \frac{1}{\tau_{0}} a_{0} \rho_{\mathrm{ns}, 0}-\frac{1}{2 \tau_{0}} \gamma_{0} a_{0}\left(E_{\mathrm{s}} a_{0}-\rho_{\mathrm{ns}, 0}\right) \\
& \times \ln \left(\frac{d_{0}}{c \tau_{0}}\right)-a_{0} \dot{\rho}_{\mathrm{ns}, 0} \ln \left(\frac{d_{0}}{c \tau_{0}}\right)
\end{aligned}
$$

respectively.
The second derivative of the correlation between the transmitted and received signals in (69) w.r.t. $\tau$, for $\tau=\tau_{0}$ and $\gamma=\gamma_{0}$, is given by

$$
\left.\frac{\partial^{2}}{\partial \tau^{2}} \rho(\tau)\right|_{\substack{\tau=\tau_{0} \\ \gamma=\gamma_{0}}}=\left.a_{0} \frac{\partial^{2}}{\partial \tau^{2}} \rho_{\mathrm{ss}}(\tau)\right|_{\tau=\tau_{0}}+\left.\frac{\partial^{2}}{\partial \tau^{2}} \rho_{\mathrm{ns}}(\tau)\right|_{\tau=\tau_{0}},
$$

where the second term is given by

$$
\left.\frac{\partial^{2}}{\partial \tau^{2}} \rho_{\mathrm{ns}}(\tau)\right|_{\tau=\tau_{0}}=\ddot{\rho}_{\mathrm{ns}, 0},
$$

and the first term can be elaborated as

$\left.\frac{\partial^{2}}{\partial \tau^{2}} \rho_{\mathrm{ss}}(\tau)\right|_{\substack{\tau=\tau_{0} \\ \gamma=\gamma_{0}}}=\left.\int_{0}^{T_{\mathrm{o}}} \Re\left(s\left(t-\tau_{0}\right) \frac{\partial^{2}}{\partial \tau^{2}} s^{*}(t-\tau)\right) \mathrm{d} t\right|_{\tau=\tau_{0}}$.

Then, applying the change of variable $t^{\prime}=t-\tau$ in (81), we obtain

$$
\begin{aligned}
\left.\frac{\partial^{2}}{\partial \tau^{2}} \rho_{\mathrm{ss}}(\tau)\right|_{\substack{\tau=\tau_{0} \\
\gamma=\gamma_{0}}} & \left.\int_{-\tau}^{T_{\mathrm{o}}-\tau} \Re\left(s\left(t^{\prime}-\left(\tau_{0}-\tau\right)\right) \frac{\partial^{2}}{\partial t^{\prime 2}} s^{*}\left(t^{\prime}\right)\right) \mathrm{d} t^{\prime}\right|_{\tau=\tau_{0}} \\
& =\int_{-\tau_{0}}^{T_{\mathrm{o}}-\tau_{0}} \Re\left(s\left(t^{\prime}\right) \frac{\partial^{2}}{\partial t^{\prime 2}} s^{*}\left(t^{\prime}\right)\right) \mathrm{d} t^{\prime} \\
& =\int_{-\tau_{0}}^{T_{\mathrm{o}}-\tau_{0}} \Re\left(s\left(t^{\prime}\right) \frac{\partial}{\partial t^{\prime}} s^{*}\left(t^{\prime}\right)\right) \mathrm{d} t^{\prime} \\
& =\int_{-\tau_{0}}^{T_{\mathrm{o}}-\tau_{0}}\left|\frac{\partial}{\partial t^{\prime}} s\left(t^{\prime}\right)\right|^{2} \mathrm{~d} t^{\prime},
\end{aligned}
$$

in which the first term vanishes, and the change of variable $t=t^{\prime}+\tau_{0}$ in the second term yields

$$
\left.\frac{\partial^{2}}{\partial \tau^{2}} \rho_{\mathrm{ss}}(\tau)\right|_{\substack{\tau=\tau_{0} \\ \gamma=\gamma_{0}}}=-\int_{0}^{T_{\mathrm{o}}}\left|\frac{\partial}{\partial t} s(t)\right|^{2} \mathrm{~d} t=-\int_{-\infty}^{\infty}\left|\frac{\partial}{\partial t} s(t)\right|^{2} \mathrm{~d} t .
$$

The expression of the effective bandwidth in the time domain is given by $\bar{\beta}=\frac{1}{2 \pi} \sqrt{\frac{\int_{-\infty}^{\infty}\left|\frac{\partial}{\partial t} s(t)\right|^{2} \mathrm{~d} t}{\int_{-\infty}^{\infty}|s(t)|^{2} \mathrm{~d} t}}$. Then, by including in (83) the expression of the effective bandwidth, we have

$$
\left.\frac{\partial^{2}}{\partial \tau^{2}} \rho_{\mathrm{sS}}(\tau)\right|_{\tau=\tau_{0}}=-4 \pi^{2} \bar{\beta}^{2} E_{\mathrm{S}}
$$

and the expression of $\left.\frac{\partial^{2}}{\partial \tau^{2}} \rho(\tau)\right|_{\substack{\tau=\tau_{0} \\ \gamma=\gamma_{0}}}$ can be written as

$$
\left.\frac{\partial^{2}}{\partial \tau^{2}} \rho(\tau)\right|_{\substack{\tau=\tau_{0} \\ \gamma=\gamma_{0}}}=-4 \pi^{2} \bar{\beta}^{2} E_{\mathrm{s}} a_{0}+\ddot{\rho}_{\mathrm{ns}, 0} .
$$


Finally, substituting (71), (76), and (85) into (67) for $\tau=$ $\tau_{0}$, we obtain

$$
\begin{aligned}
\left.\frac{\partial^{2}}{\partial \tau^{2}} f_{\mathrm{ML}}(\tau, \gamma)\right|_{\substack{\tau=\tau_{0} \\
\gamma=\gamma_{0}}} & =\frac{1}{2 \tau_{0}^{2}} \gamma_{0}^{2} E_{\mathrm{s}} a_{0}^{2}+8 \pi^{2} \bar{\beta}^{2} E_{\mathrm{s}} a_{0}^{2} \\
& -\frac{1}{\tau_{0}^{2}} \gamma_{0}\left(1+\frac{1}{2} \gamma_{0}\right) a_{0} \rho_{\mathrm{ns}, 0} \\
& +\frac{1}{\tau_{0}} 2 \gamma_{0} a_{0} \dot{\rho}_{\mathrm{ns}, 0}-2 a_{0} \ddot{\rho}_{\mathrm{ns}, 0} .
\end{aligned}
$$

In order to get the expectation w.r.t. the noise of (86), we need the expectations w.r.t. the noise of the correlation function $\rho_{\mathrm{ns}, 0}$ and its derivatives. Fortunately, it is straightforward to show that

$$
\begin{aligned}
& \mathrm{E}_{n(t)}\left\{\rho_{\mathrm{ns}, 0}\right\}=0, \\
& \mathrm{E}_{n(t)}\left\{\dot{\rho}_{\mathrm{ns}, 0}\right\}=0, \\
& \mathrm{E}_{n(t)}\left\{\ddot{\rho}_{\mathrm{ns}, 0}\right\}=0 .
\end{aligned}
$$

Thereby, the expected value of (86) w.r.t. the noise for $\tau=$ $\tau_{0}$ is given by

$$
\mathrm{E}_{n(t)}\left\{\left.\frac{\partial^{2}}{\partial \tau \partial \tau} f_{\mathrm{ML}}(\tau, \gamma)\right|_{\substack{\tau=\tau_{0} \\ \gamma=\gamma_{0}}}\right\}=\frac{1}{2 \tau_{0}^{2}} \gamma_{0}^{2} E_{\mathrm{s}} a_{0}^{2}+8 \pi^{2} \bar{\beta}^{2} E_{\mathrm{s}} a_{0}^{2} .
$$

Finally, substituting (77), (78), and (88) into (23), we obtain the final expression of the error given in Equation (27).

\section{Appendix 2: derivation of the MSE $\mathrm{E}_{n(t)}\left\{\left(\varepsilon_{\mathrm{TE}}\left(\tau_{0}, \gamma\right)\right)^{2}\right\}$} In this appendix, we derive explicitly a closed formula for the MSE of the ToA estimation based on the Taylor series expansion, i.e., we obtain a closed expression of the MSE in Equation (32). Substituting Equations (24), (25), and (26) into (32) yields

$$
\begin{aligned}
& \mathrm{E}_{n(t)}\left\{\left(\varepsilon_{T E}\left(\tau_{0}, \gamma\right)\right)^{2}\right\} \\
& \approx \frac{\mathrm{E}_{n(t)}\left\{\left(\left(\gamma-\gamma_{0}\right) \frac{1}{2 \tau_{0}} \gamma_{0} a_{0} E_{\mathrm{s}} \ln \left(\frac{d_{0}}{c \tau_{0}}\right)-\frac{1}{\tau_{0}} \gamma_{0} \rho_{\mathrm{ns}, 0}+2 \dot{\rho}_{\mathrm{ns}, 0}\right)^{2}\right\}}{\left(\frac{1}{2 \tau_{0}^{2}} \gamma_{0}^{2} E_{\mathrm{s}} a_{0}+8 \pi^{2} \bar{\beta}^{2} E_{\mathrm{s}} a_{0}\right)^{2}} \\
& =\frac{\mathrm{E}_{n(t)}\left\{\left(k_{1}+k_{2} \rho_{\mathrm{ns}, 0}+k_{3} \dot{\rho}_{\mathrm{ns}, 0}\right)^{2}\right\}}{\left(\frac{1}{2 \tau_{0}^{2}} \gamma_{0}^{2} E_{\mathrm{s}} a_{0}+8 \pi^{2} \bar{\beta}^{2} E_{\mathrm{s}} a_{0}\right)^{2}},
\end{aligned}
$$

where $k_{1}, k_{2}$, and $k_{3}$ are given by

$$
\begin{aligned}
& k_{1}=\left(\gamma-\gamma_{0}\right) \frac{1}{2 \tau_{0}} \gamma_{0} a_{0} E_{\mathrm{s}} \ln \left(\frac{d_{0}}{c \tau_{0}}\right) \\
& k_{2}=-\frac{1}{\tau_{0}} \gamma_{0}, \quad \text { and } \quad k_{3}=2 .
\end{aligned}
$$

By using (87a) and (87b), it is easily shown that the numerator in $(89)$ reduces to

$\mathrm{E}_{n(t)}\left\{\left(k_{1}-k_{2} \rho_{\mathrm{ns}, 0}+k_{3} \dot{\rho}_{\mathrm{ns}, 0}\right)^{2}\right\}=\mathrm{E}_{n(t)}\left\{k_{1}^{2}+k_{2}^{2} \rho_{\mathrm{ns}, 0}^{2}+k_{3}^{2} \dot{\rho}_{\mathrm{ns}, 0}^{2}\right\}$,

where $\mathrm{E}_{n(t)}\left\{\rho_{\mathrm{ns}, 0}^{2}\right\}, \mathrm{E}_{n(t)}\left\{\dot{\rho}_{\mathrm{ns}, 0}^{2}\right\}$, and $\mathrm{E}_{n(t)}\left\{\rho_{\mathrm{ns}, 0} \dot{\rho}_{\mathrm{ns}, 0}\right\}$ are given by

$$
\begin{aligned}
\mathrm{E}_{n(t)}\left\{\rho_{\mathrm{ns}, 0}^{2}\right\} & =\frac{1}{2} E_{\mathrm{s}} \sigma_{\mathrm{n}}^{2}, \\
\mathrm{E}_{n(t)}\left\{\dot{\rho}_{\mathrm{ns}, 0}^{2}\right\} & =2 \pi^{2} E_{\mathrm{s}} \bar{\beta}^{2} \sigma_{\mathrm{n}}^{2}, \\
\mathrm{E}_{n(t)}\left\{\rho_{\mathrm{ns}, 0} \dot{\rho}_{\mathrm{ns}, 0}\right\} & =0 .
\end{aligned}
$$

Then, substituting (93a), (93b), and (93c) into (92), we have

$$
\begin{aligned}
\mathrm{E}_{n(t)}\left\{\left(k_{1}-k_{2} \rho_{\mathrm{ns}, 0}+k_{3} \dot{\rho}_{\mathrm{ns}, 0}\right)^{2}\right\}= & k_{1}^{2}+\frac{1}{2} E_{\mathrm{s}} \sigma_{\mathrm{n}}^{2} k_{2}^{2} \\
& +2 \pi^{2} E_{\mathrm{s}} \bar{\beta}^{2} \sigma_{\mathrm{n}}^{2} k_{3}^{2},
\end{aligned}
$$

and, therefore, plugging (94) into (89), we obtain

$$
\mathrm{E}_{n(t)}\left\{\left(\varepsilon_{T E}\left(\tau_{0}, \gamma\right)\right)^{2}\right\} \approx \frac{k_{1}^{2}+\frac{1}{2} E_{\mathrm{s}} \sigma_{\mathrm{n}}^{2} k_{2}^{2}+2 \pi^{2} E_{\mathrm{s}} \bar{\beta}^{2} \sigma_{\mathrm{n}}^{2} k_{3}^{2}}{E_{\mathrm{s}}^{2} a_{0}^{2}\left(\frac{1}{2 \tau_{0}^{2}} \gamma_{0}^{2}+8 \pi^{2} \bar{\beta}^{2}\right)^{2}} .
$$

Finally, substituting the expression of $k_{1}, k_{2}$, and $k_{3}$ into (95), we obtain the final expression of the MSE, given in Equation (33).

\section{Appendix 3: derivation of the effective bandwidth of the second derivative Gaussian pulse}

In this appendix, we obtain the effective bandwidth $\bar{\beta}$ of the second derivative Gaussian pulse in Equation (64), necessary for the numerical results in Section 4.

The effective bandwidth can be derived from (see, e.g., [25])

$$
\bar{\beta}=\frac{1}{2 \pi} \sqrt{\frac{\int_{-\infty}^{\infty} \omega^{2}|S(\omega)|^{2} \mathrm{~d} \omega}{\int_{-\infty}^{\infty}|S(\omega)|^{2} \mathrm{~d} \omega}},
$$


where $S(\omega)$ is the Fourier transform of the transmitted signal $s(t)$. Consider the Fourier transform of the secondderivative monocycle with finite duration pulse from

$$
\begin{aligned}
S(\omega) & =\mathcal{F}\{s(t)\}=\int_{-\infty}^{\infty} s(t) \mathrm{e}^{-\mathrm{j} \omega t} \mathrm{~d} t \\
& =\int_{-\frac{1}{2} T_{\mathrm{p}}}^{\frac{1}{2} T_{\mathrm{p}}}\left(1-4 \pi\left(\frac{t}{\tau_{\mathrm{p}}}\right)^{2}\right) \mathrm{e}^{-2 \pi\left(\frac{t}{\tau_{\mathrm{p}}}\right)^{2}} \mathrm{e}^{-\mathrm{j} \omega t} \mathrm{~d} t,
\end{aligned}
$$

where both $S(\omega)$ and $\mathcal{F}\{s(t)\}$ are standard notation for the Fourier transform of the continuous time signal $s(t)$. Assuming that $\mathrm{e}^{-2 \pi\left(\frac{t}{\tau_{\mathrm{p}}}\right)^{2}} \approx 0$ for $|t|>\frac{1}{2} T_{\mathrm{p}}$, we can split $S(\omega)$ into two terms, namely

$$
S(\omega)=\mathcal{F}\left\{\mathrm{e}^{-\left(\frac{t}{\frac{\tau_{\mathrm{p}}}{\sqrt{2 \pi}}}\right)^{2}}\right\}-2 \mathcal{F}\left\{\left(\frac{t}{\frac{\tau_{\mathrm{p}}}{\sqrt{2 \pi}}}\right)^{2} \mathrm{e}^{-\left(\frac{t}{\frac{\tau_{\mathrm{p}}}{\sqrt{2 \pi}}}\right)^{2}}\right\} .
$$

Using the time scaling and the frequency properties of the Fourier transform in (98), we obtain

$$
S(\omega)=\left|\frac{\tau_{\mathrm{p}}}{\sqrt{2 \pi}}\right| \mathcal{F}\left\{\mathrm{e}^{-t^{2}}\right\}-2 \mathrm{j}^{2}\left|\frac{\tau_{\mathrm{p}}}{\sqrt{2 \pi}}\right| \frac{\partial^{2}}{\partial \omega^{2}} \mathcal{F}\left\{\mathrm{e}^{-t^{2}}\right\} .
$$

Since $\mathcal{F}\left\{\mathrm{e}^{-t^{2}}\right\}=\sqrt{\pi} \mathrm{e}^{-\frac{\omega^{2}}{4}},(99)$ reduces to

$$
\begin{aligned}
S(\omega) & =\sqrt{\pi}\left|\frac{\tau_{\mathrm{p}}}{\sqrt{2 \pi}}\right| \mathrm{e}^{-\frac{\omega^{2}}{4}}+2 \sqrt{\pi}\left|\frac{\tau_{\mathrm{p}}}{\sqrt{2 \pi}}\right| \frac{\partial^{2}}{\partial \omega^{2}} \mathrm{e}^{-\frac{\omega^{2}}{4}} \\
& =\frac{\tau_{\mathrm{p}}}{2 \sqrt{2}} \omega^{2} \mathrm{e}^{-\frac{\omega^{2}}{4}}
\end{aligned}
$$

In order to obtain the effective bandwidth of the transmitted signal defined by (96), we need to obtain the value of $|S(\omega)|^{2}$, which is given by

$$
|S(\omega)|^{2}=\frac{\tau_{\mathrm{p}}^{6}}{32 \pi^{2}} \omega^{4} \mathrm{e}^{-\frac{\tau_{\mathrm{p}}^{2}}{4 \pi} \omega^{2}} .
$$

Then, the integral $\int_{-\infty}^{\infty}|S(\omega)|^{2} \mathrm{~d} \omega$ can be written as

$$
\begin{aligned}
\int_{-\infty}^{\infty}|S(\omega)|^{2} \mathrm{~d} \omega & =\int_{-\infty}^{\infty} \frac{\tau_{\mathrm{p}}^{6}}{32 \pi^{2}} \omega^{4} \mathrm{e}^{-\frac{\tau_{\mathrm{p}}^{2}}{4 \pi} \omega^{2}} \mathrm{~d} \omega \\
& =\frac{\tau_{\mathrm{p}}^{6}}{16 \pi^{2}} \int_{0}^{\infty} \omega^{4} \mathrm{e}^{-\frac{\tau_{\mathrm{p}}^{2}}{4 \pi} \omega^{2}} \mathrm{~d} \omega .
\end{aligned}
$$

In (102), there is an integral with the form $\int_{0}^{\infty} x^{n} \mathrm{e}^{-\eta x^{2}} \mathrm{~d} x$. Using variable change $y=\eta x^{2}$, the integral $\int_{0}^{\infty} x^{n} \mathrm{e}^{-\eta x^{2}} \mathrm{~d} x$ may be rewritten as a function of $y$ in the following equivalent form:

$$
\begin{aligned}
\int_{0}^{\infty} x^{n} \mathrm{e}^{-\eta x^{2}} \mathrm{~d} x & =\int_{0}^{\infty}\left(x^{2}\right)^{\frac{n}{2}} \mathrm{e}^{-\eta x^{2}} \mathrm{~d} x \\
& =\frac{1}{2}\left(\frac{1}{\eta}\right)^{\frac{n+1}{2}} \int_{0}^{\infty} y^{\frac{n-1}{2}} \mathrm{e}^{-y} \mathrm{~d} y .
\end{aligned}
$$

To resolve (103), we use the Gamma function $\Gamma(m)=$ $\int_{0}^{\infty} y^{m-1} \mathrm{e}^{-y} \mathrm{~d} y$. Choosing $m=\frac{n+1}{2}$, it is readily seen that the integral in (103) can be written as

$$
\int_{0}^{\infty} x^{n} \mathrm{e}^{-\eta x^{2}} \mathrm{~d} x=\frac{1}{2}\left(\frac{1}{\eta}\right)^{\frac{n+1}{2}} \Gamma\left(\frac{n+1}{2}\right) .
$$

Thereby, by selecting $\eta=\frac{\tau_{\mathrm{p}}^{2}}{4 \pi}$ and $n=4$ and using (104), the solution of (102) is given by

$$
\int_{-\infty}^{\infty}|S(\omega)|^{2} \mathrm{~d} \omega=\frac{\tau_{\mathrm{p}}^{6}}{16 \pi^{2}}\left(\frac{4 \pi}{\tau_{\mathrm{p}}^{2}}\right)^{\frac{5}{2}} \Gamma\left(\frac{5}{2}\right) .
$$

The integral $\int_{-\infty}^{\infty} \omega^{2}|S(\omega)|^{2} \mathrm{~d} \omega$ is evaluated as follows. We start with a straightforward manipulation to obtain

$$
\begin{aligned}
\int_{-\infty}^{\infty} \omega^{2}|S(\omega)|^{2} \mathrm{~d} \omega & =\int_{-\infty}^{\infty} \frac{\tau_{\mathrm{p}}^{6}}{32 \pi^{2}} \omega^{6} \mathrm{e}^{-\frac{\tau_{\mathrm{p}}^{2}}{4 \pi} \omega^{2}} \mathrm{~d} \omega \\
& =\frac{\tau_{\mathrm{p}}^{6}}{32 \pi^{2}} 2 \int_{0}^{\infty} \omega^{6} \mathrm{e}^{-\frac{\tau_{\mathrm{p}}^{2}}{4 \pi} \omega^{2}} \mathrm{~d} \omega .
\end{aligned}
$$

Then, applying $\eta=\frac{\tau_{\mathrm{p}}^{2}}{4 \pi}$ and $n=6$, we can use (104) to arrive at the solution

$$
\int_{-\infty}^{\infty} \omega^{2}|S(\omega)|^{2} \mathrm{~d} \omega=\frac{\tau_{\mathrm{p}}^{6}}{16 \pi^{2}}\left(\frac{4 \pi}{\tau_{\mathrm{p}}^{2}}\right)^{\frac{7}{2}} \Gamma\left(\frac{7}{2}\right) .
$$

Substituting (105) and (107) into (96) yields

$$
\bar{\beta}=\frac{1}{2 \pi} \sqrt{\frac{\frac{\tau_{\mathrm{p}}^{6}}{32 \pi^{2}}\left(\frac{4 \pi}{\tau_{\mathrm{p}}^{2}}\right)^{\frac{7}{2}} \Gamma\left(\frac{7}{2}\right)}{\frac{\tau_{\mathrm{p}}^{6}}{32 \pi^{2}}\left(\frac{4 \pi}{\tau_{\mathrm{p}}^{2}}\right)^{\frac{5}{2}} \Gamma\left(\frac{5}{2}\right)} .}
$$

Using the property of the Gamma function $\Gamma(m+1)=$ $m \Gamma(m)$, the ratio of Gammas reduces to $\Gamma\left(\frac{7}{2}\right) / \Gamma\left(\frac{5}{2}\right)=\frac{5}{2}$; therefore, the expression in (108) finally reduces to

$$
\bar{\beta}=\frac{1}{\tau_{\mathrm{p}}} \sqrt{\frac{5}{2 \pi}} .
$$

\section{Appendix 4: derivation of the effective absolute central frequency of the second derivative Gaussian pulse} In this appendix, we derive the central frequency of the second derivative Gaussian pulse, i.e., the central frequency given in Equation (64), necessary for the numerical examples in Section 4. 
Since we work with a carrierless system, we need to elaborate the effective central frequency,

$$
\bar{f}=\frac{1}{2 \pi} \frac{\int_{-\infty}^{\infty} \omega|S(\omega)|^{2} \mathrm{~d} \omega}{\int_{-\infty}^{\infty}|S(\omega)|^{2} \mathrm{~d} \omega} .
$$

However, it is readily to see that the denominator of (110) is positive (see Equation (105)) while, for the numerator, we obtain

$$
\begin{aligned}
\int_{-\infty}^{\infty} \omega|S(\omega)|^{2} \mathrm{~d} \omega & =\int_{-\infty}^{+\infty} \frac{\tau_{\mathrm{p}}^{6}}{32 \pi^{2}} \omega^{5} \mathrm{e}^{-\frac{\tau_{\mathrm{p}}^{2}}{4 \pi} \omega^{2}} \mathrm{~d} \omega \\
& =\frac{\tau_{\mathrm{p}}^{6}}{32 \pi^{2}}\left(\int_{-\infty}^{0} \omega^{5} \mathrm{e}^{-\frac{\tau_{\mathrm{p}}^{2}}{4 \pi} \omega^{2}} \mathrm{~d} \omega\right. \\
& \left.+\int_{0}^{+\infty} \omega^{5} \mathrm{e}^{-\frac{\tau_{\mathrm{p}}^{2}}{4 \pi} \omega^{2}} \mathrm{~d} \omega\right)=0
\end{aligned}
$$

where we have used Equation (104). Therefore, $\bar{f}=0$ and we need to approximate the central frequency by the effective absolute central frequency defined as

$$
\bar{f}_{\mathrm{abs}}=\frac{1}{2 \pi} \frac{\int_{-\infty}^{\infty}|\omega||S(\omega)|^{2} \mathrm{~d} \omega}{\int_{-\infty}^{\infty}|S(\omega)|^{2} \mathrm{~d} \omega} .
$$

Then, we have to evaluate

$$
\begin{aligned}
\int_{-\infty}^{\infty}|\omega||S(\omega)|^{2} \mathrm{~d} \omega= & \frac{\tau_{\mathrm{p}}^{6}}{32 \pi^{2}}\left(\int_{-\infty}^{0}\left|\omega^{5}\right| \mathrm{e}^{-\frac{\tau_{\mathrm{p}}^{2}}{4 \pi} \omega^{2}} \mathrm{~d} \omega\right. \\
& \left.+\int_{0}^{+\infty} \omega^{5} \mathrm{e}^{-\frac{\tau_{\mathrm{p}}^{2}}{4 \pi} \omega^{2}} \mathrm{~d} \omega\right)
\end{aligned}
$$

It is straightforward to show that

$$
\int_{-\infty}^{\infty}|\omega||S(\omega)|^{2} \mathrm{~d} \omega=\frac{\tau_{\mathrm{p}}^{6}}{32 \pi^{2}} 2 \int_{0}^{\infty} \omega^{5} \mathrm{e}^{-\frac{\tau_{\mathrm{p}}^{2}}{4 \pi} \omega^{2}} \mathrm{~d} \omega,
$$

and applying the result of (104) into (114), with $\eta=\frac{\tau_{\mathrm{p}}^{2}}{4 \pi}$ and $n=5$, we obtain

$$
\int_{-\infty}^{\infty}|\omega||S(\omega)|^{2} \mathrm{~d} \omega=\frac{\tau_{\mathrm{p}}^{6}}{16 \pi^{2}}\left(\frac{4 \pi}{\tau_{\mathrm{p}}^{2}}\right)^{\frac{6}{2}} \Gamma\left(\frac{6}{2}\right)=8 \pi .
$$

Finally, substituting (105) and (115) into (112), we arrive at

$$
\bar{f}_{\mathrm{abs}}=\frac{1}{2 \pi} \frac{8 \pi}{\frac{3 \pi}{4} \tau_{\mathrm{p}}}=\frac{1}{\tau_{\mathrm{p}}} \frac{16}{3 \pi} .
$$

\section{Abbreviations}

$\tau$, propagation delay or ToA; $\tau_{0}$, true value of the ToA, $\gamma$, PLE; $\gamma_{0}$, true value of the PLE; $\delta_{\gamma}=\gamma-\gamma_{0}$, PLE mismatch; $s(t)$, transmitted signal; $r(t)$, received signal; $n(t)$, additive noise (zero-mean white Gaussian process with variance $\left.\sigma_{\mathrm{n}}^{2}\right) ; E_{s}$, energy of the transmitted signal; $a=a(\tau, \gamma)$, path gain in the model of Section 3.2, which is a deterministic function of the ToA $(\tau)$ and the PLE $(\gamma)$; $\psi_{\mathrm{dB}}$, shadow fading variable (assumed Gaussian distributed with zero mean and variance $\left.\sigma_{\mathrm{dB}}^{2}\right) ; a(\psi)$, path gain in the model of Section 4 , which is a random variable that depends on the shadow fading $\psi=10 \frac{\psi_{\mathrm{dB}}}{10}$ and, deterministically, on the ToA $(\tau)$ and the PLE $(\gamma) ;-f_{M L}(\tau, \gamma)$, log-likelihood function; $\rho(\tau)$, correlation between the received and the transmitted signals (random variable); $\rho_{\mathrm{ss}}(\tau)$, autocorrelation of the transmitted signal (random variable); $\rho_{n s}(\tau)$, correlation between the noise and the transmitted signal (random variable); $\hat{\tau}_{\mathrm{ML}}$ and $\hat{\gamma}_{\mathrm{ML}}$, ToA and PLE estimates that jointly maximize log-likelihood function $-f_{\mathrm{ML}}(\tau, \gamma) ; \hat{\tau}_{\mathrm{ML}}(\gamma)$, the conditional ML estimator of the ToA obtained for a fixed (but imperfect or mismatched) value of the PLE $(\gamma) ; \varepsilon_{F}\left(\hat{\tau}_{\mathrm{ML}}, \hat{\gamma}_{\mathrm{ML}}\right)$, Friedlander's error in error given in (19); $\tilde{\varepsilon}_{\mathrm{TE}}\left(\tau_{0}, \gamma\right)$, TOA estimation error given in (22), Section 3.2; $\varepsilon_{\mathrm{TE}}\left(\tau_{0}, \gamma\right)$, ToA estimation error given in (23), Section 3.2; $B\left(\tau_{0}, \gamma\right)$, theoretical approximation of the bias of the ToA estimation given in (37), Section $4.1 ; \varepsilon^{2}\left(\tau_{0}, \gamma, \psi\right)$, theoretical approximation of the MSE of the ToA estimation given in (41) in Section 4.1, which is a random variable that depends on the shadow fading $\psi$;

$\bar{\varepsilon}_{\mathrm{TE}}\left(\tau_{0}, \gamma, \psi\right)$, ToA estimation error given in (53), Section 4.2; $\bar{\varepsilon}_{\mathrm{TE}}^{(i)}\left(\tau_{0}, \gamma\right)$, sample of the ToA estimation error given in 61 , Section $4.2 ; \mu^{N}\left(\tau_{0}, \gamma\right)$, Monte-Carlo approximation of the bias of the ToA estimation given in (62), Section 4.2; $\varepsilon^{2, N}(\tau, \gamma)$, Monte-Carlo approximation of the MSE of the ToA estimation given in (63), Section 4.2; $\frac{S}{N}$, the signal-to-noise ratio (SNR).

\section{Competing interests}

The authors declare that they have no competing interests.

\section{Acknowledgements}

Isabel Valera is supported by the Plan Regional-Programas I+D of Comunidad de Madrid (AGES-CM S2010/BMD-2422). Bamrung T. Sieskul is grateful to Dr. Feng Zheng and Prof. Dr.-Ing. Thomas Kaiser for partial technical ideas and EUWB research project for financial support during the research period at the Institute of Communications Technology, Leibniz University Hannover, and to Prof. Roberto López-Valcarce for financial support during the research period at the Department of Signal Theory and Communications, University of Vigo. The authors also acknowledge the support of Ministerio de Economía y Competitividad of Spain (project DEIPRO TEC2009-14504-C02-00 and program Consolider-Ingenio 2010 CSD2008-00010 COMONSENS).

\section{Author details}

${ }^{1}$ Department of Signal Theory and Communications, University Carlos III of Madrid, Madrid, Spain. ${ }^{2}$ Signal Theory and Communications Department, University of Vigo, Vigo, Spain.

Received: 16 November 2012 Accepted: 24 May 2013 Published: 11 June 2013

\section{References}

1. S Ping, Delay Measurement Time Synchronization for Wireless Sensor Networks. (Intel Research Berkeley, Berkeley, 2003)

2. Abdulla $\mathrm{H}, \mathrm{A}$ comparative study of time-delay estimation techniques using microphone arrays. Report No. 619, Department of Electrical and Computer Engineering, The University of Auckland, New Zealand, 2005, pp 1-57

3. G Balogh, Lédeczi Á, M Maróti. Time of arrival data fusion for source localization (Budapest Hungary, 10-14 July 2005)

4. S Gezici, Z Tian, G Giannakis, H Kobayashi, A Molisch, H Poor, Z Sahinoglu, Localization via ultra-wideband radios: a look at positioning aspects for future sensor networks. IEEE Signal Process. Mag. 22(4), 70-84 (2005)

5. Y Qi, H Kobayashi, H Suda, On time-of-arrival positioning in a multipath environment. IEEE Trans. Veh. Technol. 55(5), 1516-1526 (2006)

6. V Vivekan, WWS Wong, S Member, Concentric anchor beacon localization algorithm for wireless sensor networks. IEEE Trans. Veh. Technol. 56(5), 2733-2744 (2007) 
7. J Shirahama, T Ohtsuki, in Proceedings of the IEEE vehicular technology conference. RSS-based localization in environments with different path loss exponent for each link (Marina Bay Singapore, 11-14 May 2008)

8. B Sieskul, F Zheng, T Kaiser, A hybrid SS-ToA wireless NLoS geolocation based on path attenuation: ToA estimation and CRB for mobile position estimation. IEEE Trans. Veh. Technol. 58(9), 4930-4942 (2009)

9. Y Chen, E Gunawan, KS Low, SC Wang, CB Soh, LL Thi, Time of arrival data fusion method for two-dimensional ultrawideband breast cancer detection. IEEE Trans. Antennas Propagat. 55(10), 2852-2865 (2007)

10. BT Sieskul, $F$ Zheng, T Kaiser, in Proceedings of the 10th IEEE international workshop on signal processing advances in wireless communications 2009 (SPAWC 2009). Time-of-arrival estimation in path attenuation (Perugia, Italy, 2009)

11. G Mao, BD Anderson, B Fidan, Path loss exponent estimation for wireles sensor network localization. Comput. Netw. 51(10), 2467-2483 (2007)

12. ZZ Ruisi He, J Ding, An empirical path loss model and fading analysis for high-speed railway viaduct scenarios. IEEE Antennas Wireless Propagat. Lett. 10, 808-812 (2011)

13. X Zhao, L Razoumov, LJ Greenstein, Path loss estimation algorithms and results for RF sensor networks. Proc. IEEE Veh. Tech. Conf. 7, 4593-4596 (2004)

14. S Srinivasa, M Haenggi, in Proceedings of the IEEE information theory and applications workshop (ITA '09). Path loss exponent estimation in large wireless networks (San Diego, CA, 2009)

15. TMogi, T Ohtsuki, in 14th Asia-Pacific conference on communications (APCC). ToA localization using, RSS weight with path loss exponents estimation in NLOS environments (Tokyo, Japan, 2008)

16. N Benvenuto, F Santucci, A least squares path-loss estimation approach to handover algorithms. IEEE Trans. Veh. Technol. 48(2), 437-447 (1999)

17. CD Richmond, in Proceedings of the fourth IEEE workshop on sensor array and multichannel processing (SAM). On the threshold region mean-squared error performance of maximum-likelihood direction-of arrival estimation in the presence of signal model mismatch (Waltham, MA, 2006)

18. B Friedlander, Sensitivity analysis of the maximum likelihood direction-finding algorithm. IEEE Trans. Aerosp. Electron. Syst. 26(6), 953-968 (1990)

19. A Goldsmith, Wireless Communications. (Cambridge University Press, London, UK, 2005)

20. Y Qi, H Kobayashi, H Suda, Analysis of wireless geolocation in a non-line-of-sight environment. IEEE Trans. Wireless Commun. 5(3), 672-681 (2006)

21. D Slepian, Estimation of signal parameters in the presences of noise. IRE Trans. Inform. Theory. 3, 68-89 (1954)

22. P Swerling, Parameter estimation for waveforms in additive Gaussian noise. J. Soc. Indust. Appl. Math. 7(2), 152-166 (1959)

23. H Urkowitz, Signal Theory and Random Processes. (Artech House, Washington, MA, 1983)

24. HV Poor, An Introduction to, Signal Detection and Estimation, 2nd edn. (Springer, New York, 1994)

25. CW Helstrom, Elements of, Signal Detection and Estimation. (Prentice Hall, Englewood Cliffs, NJ, 1995)

26. RN McDonough, AD Whalen, Detection of, Signals in Noise, 2nd edn. (Academic, New York, 1995)

27. HL Van Trees, Detection, Estimation, and Modulation, Theory, Part I Detection, Estimation, and Linear Modulation Theory. (Wiley, New York, 2001)

28. RD Hippenstiel, Detection theory: Applications and Digital Signal Processing. (CRC Press, Boca Raton, Florida, 2002)

29. BC Levy, Principles of, Signal Detection and Parameter Estimation. (Springer, New York, 2008)

30. TS Rappaport, Wireless Communications: Principles and, Practice, 2nd edn (Prentice-Hall, Englewood Cliffs, 2002)

31. K Sharman, T Durrani, M Wax, T Kailath, in Proceedings of the IEEE conference on acoustics, speech, and signal processing (ICASSP). Asymptotic performance of eigenstructure spectral analysis methods (San Diego, CA, 1984)

32. P Stoica, A Nehorai, MUSIC, maximum likelihood and Cramér-Rao bound. IEEE Trans. Acoust., Speech, Signal Process. 37(5), 720-741 (1989)

33. M Viberg, B Ottersten, Sensor array processing based on subspace fitting. IEEE Trans. Signal Process. 39(5), 1110-1121 (1991)

34. JAC Brown, J Aitchison, The Lognormal Distribution. (Cambridge University Press, UK, 1963)
35. BT Sieskul, NLoS Localization and UWB Channel Capacity Analysis (Hannoversche Beiträge zur Nachrichtentechnik / 2: Kommunikationsnetze) (Shaker, Germany, 2010)

36. CP Robert, G Casella, Monte Carlo statistical methods. (Springer, New York, 2004)

37. Z Sahinoglu, S Gezici, I Guvenc, Ultra-Wideband Positioning, Systems Theoretical Limits, Ranging Algorithms, and Protocols. (Cambridge University Press, UK, 2008)

38. S Ghassemzadeh, R Jana, C Rice, W Turin, V Tarokh, Measurement and modeling of an ultra-wide bandwidth indoor channel. Commun. IEEE Trans. 52(10), 1786-1796 (2004)

39. MZ Win, RA Scholtz, Ultra-wide bandwidth time-hopping spread-spectrum impulse radio for wireless multiple access communications. IEEE Trans. Commun. 48(4), 679-691 (2000)

40. U Mengali, Synchronization Techniques for Digital Receivers. (Springer, New York, 1997)

doi:10.1186/1687-1499-2013-158

Cite this article as: Valera et al:: On the maximum likelihood estimation of the ToA under an imperfect path loss exponent. EURASIP Journal on Wireless Communications and Networking 2013 2013:158.

\section{Submit your manuscript to a SpringerOpen ${ }^{\circ}$ journal and benefit from:}

- Convenient online submission

- Rigorous peer review

- Immediate publication on acceptance

- Open access: articles freely available online

- High visibility within the field

- Retaining the copyright to your article

Submit your next manuscript at $>$ springeropen.com 